\title{
Tea consumption and the risk of five major cancers: a dose-response meta-analysis of prospective studies
}

\author{
Feifei $\mathrm{Yu}^{1 \dagger}$, Zhichao $\mathrm{Jin}^{1 \dagger}$, Hong Jiang ${ }^{1 \dagger}$, Chun Xiang ${ }^{1 \dagger}$, Jianyuan Tang ${ }^{2}$, Tuo $\mathrm{Li}^{3}$ and Jia He ${ }^{1 *}$
}

\begin{abstract}
Background: We conducted a dose-response meta-analysis of prospective studies to summarize evidence of the association between tea consumption and the risk of breast, colorectal, liver, prostate, and stomach cancer.

Methods: We searched PubMed and two other databases. Prospective studies that reported risk ratios (RRs) with 95\% confidence intervals (Cls) of cancer risk for $\geq 3$ categories of tea consumption were included. We estimated an overall RR with $95 \% \mathrm{Cl}$ for an increase of three cups/day of tea consumption, and, usingrestricted cubic splines, we examined a nonlinear association between tea consumption and cancer risk.
\end{abstract}

Results: Forty-one prospective studies, with a total of 3,027,702 participants and 49,103 cancer cases, were included. From the pooled overall RRs, no inverse association between tea consumption and risk of five major cancers was observed. However, subgroup analysis showed that increase in consumption of three cups of black tea per day was a significant risk factor for breast cancer (RR, 1.18; 95\% Cl, 1.05-1.32).

Conclusion: Ourresults did not show a protective role of tea in five major cancers. Additional large prospective cohort studies are needed to make a convincing case for associations.

Keywords: Tea consumption, Dose-response, Meta-analysis, Cancer

\section{Background}

Tea is a popular beverage consumed worldwide, generally in the forms of black and green tea. Tea is produced from the leaves of the Camellia sinensis plant through several processes. Black tea is the main tea beverage in the United States, Europe, and Western Asia, while green tea is more popular in China, Japan, and Korea [1]. Extensive laboratory studies using multiple animal models have suggested that tea and tea polyphenols might have an inverse association with cancer through its apoptosis-inducing, antimutagenic, and antioxidant properties [2,3].

In some recent reviews, researchers have suggested that green tea, which contains abundant polyphenols and catechins, specifically epigallocatechin-3-gallate (EGCG) 5, might have a protective effect against cancers. Using multiple approaches, studies have shown that polyphenols,

\footnotetext{
* Correspondence: hejia63@yeah.net

${ }^{\dagger}$ Equal contributors

'Department of Health Statistics, Second Military Medical University, 800

Xiangyin Road, Shanghai 200433, China

Full list of author information is available at the end of the article
}

theaflavins (TF) and thearubigins (TR) in black tea might possess chemopreventive properties. However, most of the evidence showing a protective effect of tea on cancer has been generated in animal experiments but has not been demonstrated in human trials $[4,5]$.

The World Cancer Research Fund report of 2007 concluded that the evidence for associations between the consumption of tea and risk of some major cancers was still limited and inconsistent [6]. The results from a few clinical trials and epidemiological studies also indicated that the preventive effect of tea or its extract on cancer is controversial. In a recent clinical trial evaluating the efficacy of green tea extract (GTE) on prostate cancer, it was found that the GTE had minimal clinical activity $[7,8]$. However, another Phase II clinical trial suggested that higher doses of GTE might improve the short-term outcome in patients with a higher risk of oral premalignant lesions [9]. In a cohort study conducted in the USA, tea consumption was found to have no inverse association with colorectal cancer, and the hazard ratio 
(HR) changed only slightly as tea consumption increased [10]. However, in another cohort study conducted in China, results showed that regular green tea consumption was associated with a reduced risk of colorectal cancer in smokers [11]. Some recent systematic reviews have revealed conflicting results between meta-analyses with prospective studies and those with retrospective studies [12-16].

Previous meta-analyses mainly focused on the relationship between the highest tea consumption level and either the lowest tea consumption level or non-drinkers. However, the range of tea consumption and the cut-offs for the categories differed between studies. Another drawback of the previous meta-analyses is the inclusion of retrospective case-control studies, which were sensitive to confounding variables and bias, especially recall bias. To quantitatively assess the relationship between tea consumption and risk of five major cancers, we conducted a systematic review and dose-response meta-analysis with prospective studies. The five major cancers we studied were liver, stomach, breast, prostate, and colorectal cancer.

\section{Methods}

\section{Literature search}

We performed a systematic literature search in PubMed, Embase, and Cochrane Library with a combination of the following terms: tea AND (breast OR prostate OR stomach OR gastric OR colorectal OR colorectum OR rectal OR rectum OR colon OR large bowel OR liver OR hepatic OR hepatoma) AND (cancer OR cancers OR carcinoma OR carcinomas OR neoplasm OR neoplasms). No language restrictions were imposed. Reference lists of the identified publications were also reviewed for inclusion/exclusion. We also searched the conference abstract on the website of American Society of Clinical Oncology (ASCO) annual meeting from 2004 to 2013. Two reviewers (FY and CX) independently selected studies based on the titles and abstracts of the retrieved studies. Studies were included if they met the following criteria: (1) a prospective study assessing the association between tea consumption and at least one of the five selected cancers (breast, stomach, colorectal, liver and prostate cancer); (2) a study considering at least three levels of tea consumption and providing a sample size for cases and non-cases in each exposure category [17]; and (3) a study reporting the relative risks (RRs) of different dose categories with 95\% confidence intervals (95\% CIs) adjusted for sex, age, or other factors. We excluded retrospective case-control studies because of their inherent limitations, especially recall bias. However, nested case-control studies and case-cohort studies were included in our meta-analysis because the at-risk study populations in each of the exposure categories are derived from cohort studies and diet exposure would have been investigated years before the onset of cancer, which would technically eliminate recall bias. We also excluded studies that reported total tea consumption on monthly, weekly, or daily basis, but did not provide data on number of cups or times per month, week or day. One study assessing breast cancer risk was excluded because we could not extract data stratified by sex in both exposed and unexposed groups [18]. Another study was excluded because it reported stomach cancer risk based on iced tea and hot tea consumption, but no other study provided such data [19].

\section{Data extraction}

Data extraction was performed according to the MOOSE (meta-analysis of observation studies in epidemiology) guidelines [20] (see the Additional file 1) by two reviewers (FY and CX), and verified independently for accuracy by a third reviewer (ZJ). Discrepancies were discussed with a fourth reviewer $(\mathrm{JH})$ to reach a consensus. For each included study, the following data was extracted: the title and author of the study, publication year, study population, study location, sample size, proportion of males, median of follow-up time, the type of consumed tea, covariates controlled for by matching or multivariable analysis, the numbers of cases/non-cases, total person-years, relative risk (RR) of the different exposure categories and the corresponding 95\% confidence intervals (95\% CIs), response rate, and how exposure were assessed. For studies that reported several multivariable adjusted RRs, we selected the effect estimate that adjusted for the maximum potential confounders. The quality of the included studies was assessed according to the 9-star Newcastle-Ottawa Scale (NOS) [21] by two investigators (FY and $\mathrm{HJ}$ ).

\section{Statistical analysis}

We performed a two-stage dose-response meta-analysis to examine the relationship between tea consumption and five major cancer risks. First, we synthesized the RRs across categories of tea consumption in each study [22,23]. Because the absolute risk of cancer is low, the odds ratios (ORs) in nested case-control studies approximated the RRs [24]. Pooling of RRs from each study requires the exposure levels and distribution of cases and person-years or non-cases in each category of tea consumption. However, not all studies reported the distributions of cases and person-years or non-cases for exposure categories. Nine studies did not report individuals or person-years for each category and instead reported the total sample size [10,11,25-31]. We estimated the distribution of cases for each category in such studies by using the methods described by Aune [32]. To assess exposure levels, we converted all measures into cups per day and defined $125 \mathrm{~mL}$ of tea as one cup regardless of tea type unless it was well established in a specific study population or a geographical area. If the study reported tea 
consumption as number of times, we regarded one time as one cup. As some Chinese studies reported the amount of tea leaves consumed as the measure of tea consumption, we regarded consumption of $150 \mathrm{~g}$ of tea leaves per month as one cup per day; this allowed us to universalize all of the included studies in a single standard unit [33].

If a study did not report the median of the exposure category, we assigned the level of tea consumption to categories based on the calculated midpoint of tea consumption. When the highest category was open-ended, we assumed the dose as 1.2 times the lowest bound of this category [17]. In studies reporting tea consumption by cups per month or cups per week, we redefined these exposure categories as cups per day by multiplying with $1 / 30$ or $1 / 7$, respectively. Subsequently, we estimated the overall RR by combining the RRs derived from the first step. A fixed effect model was used if there was no evidence of heterogeneity; otherwise a random effect model was adopted $[34,35]$. Forest plots were used to visually assess the RR estimates and corresponding 95\% CIs. We also tested the nonlinear relationship between tea consumption and cancer risk by modeling tea consumption levels by using restricted cubic splines with 3 knots at fixed percentiles $(10 \%, 50 \%$, and $90 \%)$ of the distribution as described by Larsson and Orsini [36,37]. A $P$ value for nonlinearity was calculated by testing the null hypothesis that the coefficient of the second and third spline was equal to zero.

Eight studies on colorectal cancer [10,26,27,31,38-41] and three studies on stomach cancer $[27,42,43]$ reported cancer risk by sex. Ten studies reported colorectal cancer risk stratified as colon or rectal cancer $[10,18,26,27,38-41,44,45]$. One study reported colon cancer risk as a distal or proximal cancer [38]. The study by Inoue et al. pooled data from six cohort Japanese studies that studied the relationship between tea consumption and stomach cancer [43]. Results from these studies were first pooled by using a fixed model and then included in the overall risk estimate. The $\chi^{2}$ test and $\mathrm{I}^{2}$ statistic were used to explore the heterogeneity among studies [46]. The Egger's regression test, Begg's rank correlation test, and visual inspection of a funnel plot were performed to assess publication bias $[47,48]$. As a rule of thumb, tests for asymmetry should be used only when there are at least 10 studies included in a meta-analysis [35]. We conducted subgroup analyses stratified by sex, tea types, and geographic regions. For breast cancer, we also performed a subgroup analysis stratified by menopausal status. We performed a sensitivity analysis in which one study at a time was removed and the rest analyzed to evaluate whether the results could have been affected markedly by a single study. To detect whether different assessment ways may bias the results, further subgroup analyses were performed by excluding studies that reported tea consumption by frequency (times/servings) [18,42,49-52], weight of tea leaves (grams) [11,27,33], or volume $(\mathrm{mL})$ $[53,54]$, rather than cups.

We used Stata (Version 12.0; Stata Corp, College Station, TX) for all analyses and all statistical tests were two-sided. $\mathrm{P}<0.05$ was considered statistically significant.

\section{Result}

\section{Study characteristics}

As of December 28, 2013, 1,881 records were retrieved by using our search strategy. After reviewing the titles and abstracts, we excluded 1,668 articles and 213 articles were further evaluated by reviewing the full texts. Finally, we identified 41 articles assessing tea consumption and cancer risk, which satisfied the inclusion criteria for our meta-analysis. A flow diagram of study selection is provided as Figure 1. Among the 41 articles, 15 assessed the relationship between tea drinking and the incidence for breast cancer [25,33,41,49,51,54-63], 15 for colorectal cancer $[10,11,18,26-29,31,38-41,44,45,59]$, 4 for liver cancer $[18,27,64,65], 7$ for prostate cancer [30,50,53,66-69], and 5 for stomach cancer $[27,42,43,52,59]$. The 41 articles included had 3,027,702 participants and 49,103 cancer cases. The cancer cases included 20,500 breast cancer patients; 16,202 colorectal cancer patients; 882 liver cancer patients; 4,698 prostate cancer patients; and 6,821 stomach cancer patients. Most of the included studies awarded more than 7 stars according to the Newcastle-Ottawa Scale and were identified as high quality. The general characteristics of the included studies are presented in Table 1.

\section{Tea consumption and cancers}

The associations between tea consumption and the risk of major cancers are shown in Figures 2, 3, 4, 5 and 6. For breast cancer, the overall RR for three cups increment per day of tea consumption was 1.02 (95\% CI, 0.98 to 1.05$)$ with mild heterogeneity among studies $(P=0.22$, $\left.I^{2}=21.2 \%\right)$. For colorectal cancer, the pooled RR for three cups increment per day was 0.98 (95\% CI, 0.93 to $1.03)$ with mild heterogeneity $\left(P=0.29, I^{2}=15.0 \%\right)$. For liver cancer, the overall RR for three cups increment per day was 0.91 (95\% CI, 0.74-1.12) with moderate heterogeneity $\left(P=0.10, I^{2}=52.5 \%\right)$. For prostate cancer, the overall RR for three cups increment per day was 1.02 (95\% CI, 0.96 to 1.09 ) with moderate heterogeneity $(P=$ $\left.0.14, I^{2}=37.8 \%\right)$. For stomach cancer, the overall RR for three cups increment per day was 0.98 (95\% CI, 0.931.03) with moderate heterogeneity $\left(P=0.15, I^{2}=40.6 \%\right)$.

As shown by Figure 7 and the P-value for nonlinearity, we found no evidence of nonlinear relationships between tea consumption and risk of cancers.

In the subgroup analysis, we pooled the studies into groups by sex, tea type, geographic region, and menopausal 


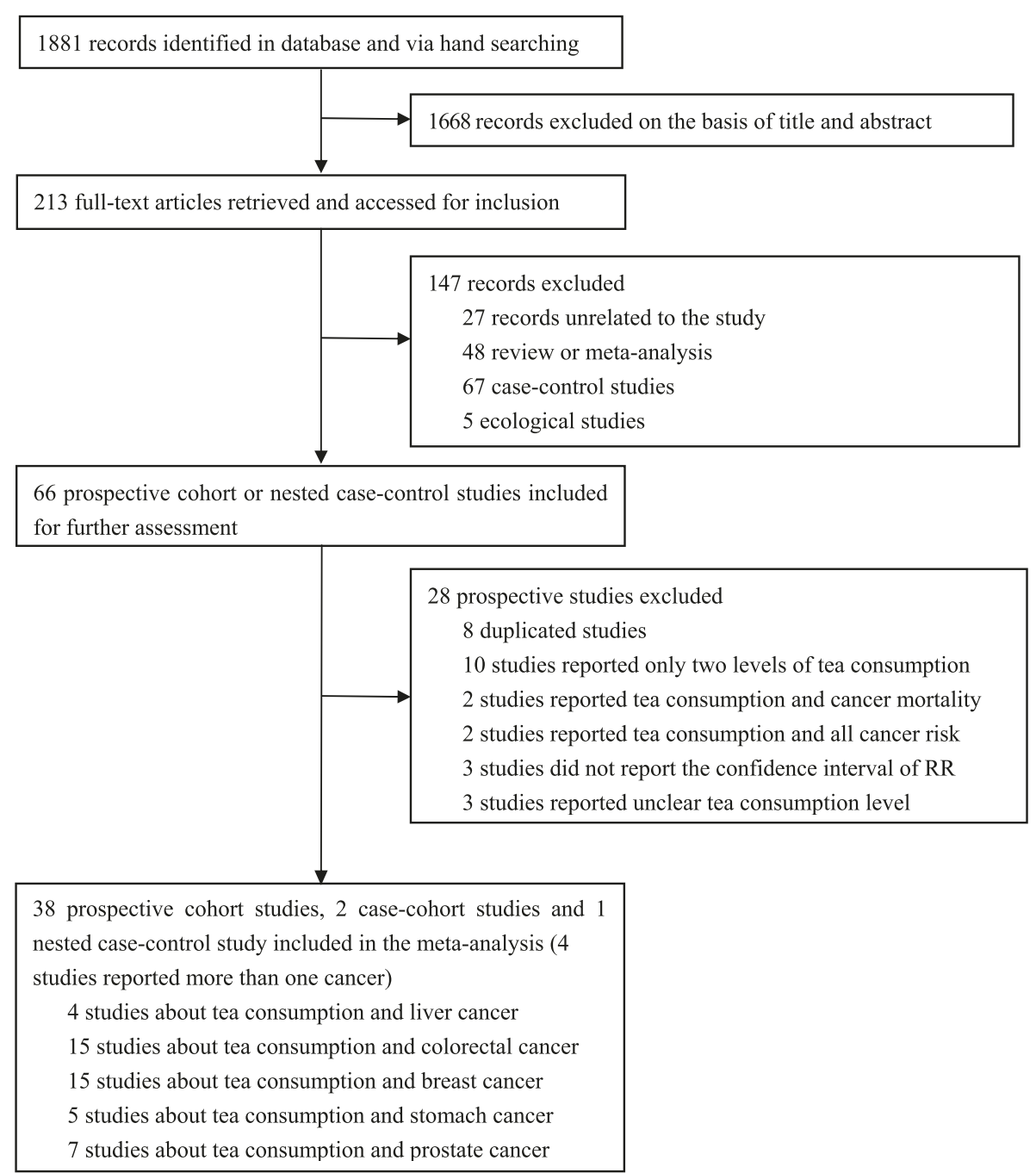

Figure 1 Summary of article selection process.

status for breast cancer. The results are shown in Table 2. We found that three cups of black tea consumption increment per day may be a risk factor for breast cancer (RR, 1.18; 95\% CI, 1.05-1.32). The result of the subgroup analysis of stomach cancer indicated that tea consumption was a preventive factor (RR, 0.88; 95\% CI, 0.80-0.98) in women. However, only three studies were included in this subgroup.

A sensitivity analysis omitting one study at a time and calculating the pooled RRs for the remainder of the studies suggested that no single study dramatically influenced the pooled RRs (results are not shown). After removing the studies that did not report tea consumption as cups per day, the results did not change significantly. On excluding a study by Inoue et al. [43], which had a significantly larger sample size in comparison with other included studies, no significant differences were observed.
Egger's regression test and Begg's rank correlation test showed no significant asymmetry of the funnel plot for breast $(P=0.59$ and $P=0.60$, respectively) and colorectal cancer $(P=0.59$ and $P=0.73$, respectively), indicating no evidence of substantial publication bias (Figure 8). For the other three types of cancer, we did not perform an analysis for publication bias because of limited numbers of included studies (no more than ten).

\section{Discussion}

The findings from our meta-analysis reveal no appreciable association between tea consumption and the relative risk of liver, stomach, breast, prostate, or colorectal cancers. The risk differences were all near zero for the five major cancers with an increase in tea consumption of three cups per day (approximately $375 \mathrm{~mL}$ per day). Subgroup analyses, stratified by sex, geographic 
Table 1 Main characteristics of the studies on tea consumption and five selected cancer included in the meta-analysis

\begin{tabular}{|c|c|c|c|c|c|c|c|c|c|}
\hline Reference & Country & Study design & Sampling & Tea type & $\begin{array}{l}\text { Number of } \\
\text { participants }\end{array}$ & $\begin{array}{l}\text { Number } \\
\text { of cases }\end{array}$ & Age & $\begin{array}{c}\text { Follow-up } \\
\text { (year) }\end{array}$ & $\begin{array}{c}\text { Male } \\
(\%)\end{array}$ \\
\hline \multicolumn{10}{|c|}{ Breast Cancer } \\
\hline $\begin{array}{l}\text { Fagherazzi et al. } \\
2011[57]\end{array}$ & France & Cohort & Population based (E3N study) & Tea (unclear) & 67703 & 2868 & $40-65$ & 11 & $0(0)$ \\
\hline $\begin{array}{l}\text { Iwasaki et al. } \\
2010[60]\end{array}$ & Japan & Cohort & Population based (JPHC study) & $\begin{array}{l}\text { Green tea \& } \\
\text { Black tea }\end{array}$ & 53793 & 845 & $40-69$ & 13.6 & $0(0)$ \\
\hline $\begin{array}{l}\text { Dai et al. } \\
2010 \text { [33] }\end{array}$ & China & Cohort & Population based (SWHS study) & Green tea & 72861 & 614 & $40-70$ & 7.3 & $0(0)$ \\
\hline $\begin{array}{l}\text { Boggs et al. } \\
2010[56]\end{array}$ & USA & Cohort & Population based (BWHS study) & Tea (unclear) & 52062 & 1268 & $21-69$ & 12 & $0(0)$ \\
\hline $\begin{array}{l}\text { Pathy et al. } \\
2010[55]\end{array}$ & Dutch & Cohort & Population based (EPIC-NL study) & Tea (unclear) & 27323 & 681 & $20-70$ & 9.6 & $0(0)$ \\
\hline $\begin{array}{l}\text { Larsson et al. } \\
2009[61]\end{array}$ & Sweden & Cohort & Population based (Swedish Mammography Cohort) & Black tea & 66651 & 2952 & $40-76$ & 17.4 & $0(0)$ \\
\hline $\begin{array}{l}\text { Ishitani et al. } \\
2008[25]\end{array}$ & USA & Cohort & Population based (Women's Health Study) & Tea (unclear) & 38432 & 1188 & $>45$ & 10 & $0(0)$ \\
\hline $\begin{array}{l}\text { Ganmaa et al. } \\
2008[58]\end{array}$ & USA & Cohort & Registered nurses (Nurses' Health Study) & Tea (unclear) & 85987 & 5272 & $30-55$ & 22 & $0(0)$ \\
\hline $\begin{array}{l}\text { Hirvonen et al. } \\
2006[54]\end{array}$ & France & Cohort & $\begin{array}{c}\text { Double-blind placebo-controlled primary-prevention } \\
\text { trial (SU.VI.MAX Study) }\end{array}$ & Tea (unclear) & 4396 & 95 & $35-60$ & 6.6 & $0(0)$ \\
\hline $\begin{array}{l}\text { Adebamowo } \\
\text { et al. } 2005[49]\end{array}$ & USA & Cohort & Registered nurses (Nurses' Health Study II) & Tea (unclear) & 90638 & 710 & $25-46$ & 4 & $0(0)$ \\
\hline $\begin{array}{l}\text { Suzuki et al. } \\
2004 \text { [63] }\end{array}$ & Japan & Cohort & Population based & Green tea & 35004 & 222 & $40-64$ & $7-9$ & $0(0)$ \\
\hline $\begin{array}{l}\text { Michels et al. } \\
2002[62]\end{array}$ & Sweden & Cohort & $\begin{array}{c}\text { Population based (Swedish Mammography Screening } \\
\text { Cohort) }\end{array}$ & Tea (unclear) & 59036 & 1271 & $40-76$ & 10.8 & $0(0)$ \\
\hline $\begin{array}{l}\text { Key et al. } \\
1999[51]\end{array}$ & Japan & Cohort & Hiroshima or Nagasaki bombings survivor (LSS study) & $\begin{array}{l}\text { Green tea \& } \\
\text { Black tea }\end{array}$ & 34759 & 405 & $\begin{array}{l}<40 \text { to } \\
>80\end{array}$ & 1969-1993 & $0(0)$ \\
\hline $\begin{array}{l}\text { Zheng et al. } \\
1996[41]\end{array}$ & USA & Cohort & Population based (lowa Women'sHealth Study) & $\begin{array}{l}\text { Non-herbal } \\
\text { tea }\end{array}$ & 35369 & 1602 & $55-69$ & 8 & $0(0)$ \\
\hline $\begin{array}{l}\text { Goldbohm } \\
\text { et al. } 1996 \text { [59] }\end{array}$ & Netherlands & Case-cohort & $\begin{array}{l}\text { Population based (Netherlands Cohort Study on Diet } \\
\text { and Cancer) }\end{array}$ & Black tea & 1376 & 507 & $55-69$ & 4.3 & $0(0)$ \\
\hline \multicolumn{10}{|c|}{ Colorectal Cancer } \\
\hline $\begin{array}{l}\text { Dominianni } \\
\text { et al. } 2013 \text { [29] }\end{array}$ & USA & Cohort & Population based (The PLCO Cancer Screening Trial) & Tea & 57398 & 683 & $55-74$ & 11.4 & $\begin{array}{l}27596 \\
(48.1)\end{array}$ \\
\hline $\begin{array}{l}\text { Sinha et al. } \\
2012[10]\end{array}$ & USA & Cohort & Population based (NIH-AARP Study) & Tea (unclear) & 489706 & 6946 & $50-71$ & 10.5 & $\begin{array}{c}292211 \\
(59.7)\end{array}$ \\
\hline $\begin{array}{l}\text { Yang et al. } \\
2011 \text { [11] }\end{array}$ & China & Cohort & Population based (SMHS Study) & Green tea & 60567 & 243 & $40-74$ & 4.6 & $\begin{array}{c}60567 \\
(100)\end{array}$ \\
\hline
\end{tabular}


Table 1 Main characteristics of the studies on tea consumption and five selected cancer included in the meta-analysis (Continued)

\begin{tabular}{|c|c|c|c|c|c|c|c|c|c|}
\hline $\begin{array}{l}\text { Simons et al. } \\
2010[38]\end{array}$ & Netherlands & Case-cohort & Population based (NLCS Study) & Tea (unclear) & 3877 & 2199 & $55-69$ & 13.3 & $\begin{array}{l}58279 \\
(48.2)\end{array}$ \\
\hline $\begin{array}{l}\text { Lee et al. } \\
2007 \text { [26] }\end{array}$ & Japan & Cohort & Population based (JPHC study) & Green tea & 96162 & 1163 & 52.1 & 10 & $\begin{array}{l}46023 \\
(47.9)\end{array}$ \\
\hline $\begin{array}{l}\text { Oba et al. } \\
2006[31]\end{array}$ & Japan & Cohort & Population based (Cohort in Takayama) & Green tea & 30221 & 213 & $>35$ & $1992-2000$ & $\begin{array}{l}13894 \\
(46.0)\end{array}$ \\
\hline $\begin{array}{l}\text { Michels et al. } \\
2005 \text { [39] }\end{array}$ & USA & Cohort & $\begin{array}{l}\text { Registered nurses and health professionals (NHS and } \\
\text { HPFS) }\end{array}$ & Tea (unclear) & 133893 & 1402 & $30-75$ & 18 and 12 & $\begin{array}{r}46099 \\
(34.4)\end{array}$ \\
\hline $\begin{array}{l}\text { Suzuki et al. } \\
2005[44]\end{array}$ & Japan & Cohort & Population based & Green tea & 26311 & 269 & $40-64$ & $8-9$ & - \\
\hline $\begin{array}{l}\text { Su et al. } \\
2002[28]\end{array}$ & USA & Cohort & Population based (NHEFS study) & Tea (unclear) & 10011 & 219 & $25-74$ & 20 & - \\
\hline $\begin{array}{l}\text { Terry et al. } \\
2001[45]\end{array}$ & Sweden & Cohort & $\begin{array}{l}\text { Population based (The Swedish Mammography } \\
\text { Screening Cohort) }\end{array}$ & Black tea & 61463 & 460 & $40-76$ & 9.6 & $0(0)$ \\
\hline $\begin{array}{l}\text { Nagano et al. } \\
2001[18]\end{array}$ & Japan & Cohort & Atomic bomb survivor (LSS study) & Green tea & 38540 & 596 & 55.3 & 16 & $\begin{array}{l}14873 \\
(38.6)\end{array}$ \\
\hline $\begin{array}{l}\text { Hartman et al. } \\
1998[40]\end{array}$ & Finnish & $\mathrm{RCT}$ & $\begin{array}{c}\text { Randomized, double-blind, placebo-controlled } \\
\text { prevention trial (ATBC Study) }\end{array}$ & Tea (unclear) & 27029 & 185 & 57.2 & 6.1 & $\begin{array}{r}27111 \\
(100)\end{array}$ \\
\hline $\begin{array}{l}\text { Zheng et al. } \\
1996[41]\end{array}$ & USA & Cohort & Population based (lowa Women's Health Study) & $\begin{array}{l}\text { Non-herbal } \\
\text { tea }\end{array}$ & 35369 & 474 & $55-69$ & 8 & $0(0)$ \\
\hline $\begin{array}{l}\text { Goldbohm } \\
\text { et al. } 1996 \text { [59] }\end{array}$ & Netherlands & Case-cohort & $\begin{array}{l}\text { Population based (Netherlands Cohort Study on Diet } \\
\text { and Cancer) }\end{array}$ & Black tea & 2929 & 564 & $55-69$ & 4.3 & $0(0)$ \\
\hline $\begin{array}{l}\text { Nechuta et al. } \\
2012[27]\end{array}$ & China & Cohort & Population based (Shanghai Women's Health Study) & Tea (any) & 69310 & 586 & $40-70$ & 11 & $0(0)$ \\
\hline \multicolumn{10}{|c|}{ Liver Cancer } \\
\hline $\begin{array}{l}\text { Nechuta et al. } \\
2012[27]\end{array}$ & China & Cohort & Population based (Shanghai Women's Health Study) & Tea (any) & 69310 & 134 & $40-70$ & 11 & $0(0)$ \\
\hline $\begin{array}{l}\text { Ui et al. } \\
2009[65]\end{array}$ & Japan & Cohort & Population based (Ohsaki Cohort study) & Green tea & 41761 & 247 & $40-79$ & 9 & $\begin{array}{l}19748 \\
(47.3)\end{array}$ \\
\hline $\begin{array}{l}\text { Inoue et al. } \\
2009[64]\end{array}$ & Japan & Cohort & $\begin{array}{c}\text { Population based (Japan Public Health Center-Based } \\
\text { Prospective Study Cohort II) }\end{array}$ & Green tea & 18815 & 110 & $40-69$ & 12.7 & $\begin{array}{l}6420 \\
(34.1)\end{array}$ \\
\hline $\begin{array}{l}\text { Nagano et al. } \\
2001[18]\end{array}$ & Japan & Cohort & Atomic bomb survivor (LSS study) & Green tea & 38540 & 391 & 55.3 & 16 & $\begin{array}{l}14873 \\
(38.6)\end{array}$ \\
\hline \multicolumn{10}{|c|}{ Prostate Cancer } \\
\hline $\begin{array}{l}\text { Geybels et al. } \\
2013 \text { [69] }\end{array}$ & Netherlands & Case-cohort & Population based (The Netherlands Cohort Study) & Black tea & 5490 & 3362 & $55-69$ & 17.3 & $\begin{array}{l}5490 \\
(100)\end{array}$ \\
\hline $\begin{array}{l}\text { Montague et al. } \\
2012 \text { [30] }\end{array}$ & Singepore & Cohort & Population based (Singapore Chinese Health Study) & $\begin{array}{l}\text { Green tea \& } \\
\text { Black tea }\end{array}$ & 27293 & 298 & $45-74$ & 11.2 & $\begin{array}{l}27293 \\
(100)\end{array}$ \\
\hline $\begin{array}{l}\text { Shafique et al. } \\
2012 \text { [68] }\end{array}$ & Canada & Cohort & $\begin{array}{l}\text { Employed men and women } \\
\text { (Collaborative Cohort Study) }\end{array}$ & Tea (unclear) & 6016 & 186 & $21-75$ & 28 & $\begin{array}{l}6016 \\
(100)\end{array}$ \\
\hline
\end{tabular}


Table 1 Main characteristics of the studies on tea consumption and five selected cancer included in the meta-analysis (Continued)

\begin{tabular}{|c|c|c|c|c|c|c|c|c|c|}
\hline $\begin{array}{l}\text { Kurahashi et al. } \\
2008[67]\end{array}$ & Japan & Cohort & Population based (Singapore Chinese Health Study) & Green tea & 49920 & 404 & $40-69$ & 15 & $\begin{array}{l}49920 \\
(100)\end{array}$ \\
\hline $\begin{array}{l}\text { Kikuchi et al. } \\
2006[66]\end{array}$ & Japan & Cohort & Population based (Ohsaki Cohort Study) & Green tea & 18961 & 110 & $40-79$ & 7 & $\begin{array}{r}18961 \\
(100)\end{array}$ \\
\hline $\begin{array}{l}\text { Allen et al. } \\
2004 \text { [50] }\end{array}$ & Japan & Cohort & Atomic-Bomb Survivors (LSS Study) & $\begin{array}{l}\text { Green tea \& } \\
\text { Black tea }\end{array}$ & 18115 & 193 & 18-99 & 16.9 & $\begin{array}{c}18115 \\
(100)\end{array}$ \\
\hline $\begin{array}{l}\text { Ellision et al. } \\
2000 \text { [53] }\end{array}$ & Canada & Cohort & Population based (NCS Study) & Tea (unclear) & 3400 & 145 & $50-84$ & 20 & $\begin{array}{l}3400 \\
(100)\end{array}$ \\
\hline \multicolumn{10}{|c|}{ Stomach Cancer } \\
\hline $\begin{array}{l}\text { Nechuta et al. } \\
2012 \text { [27] }\end{array}$ & China & Cohort & Population based (Shanghai Women's Health Study) & Tea (any) & 69310 & 293 & $40-70$ & 11 & $0(0)$ \\
\hline $\begin{array}{l}\text { Inoue et al. } \\
2009 \text { [43] }\end{array}$ & Japan & Cohort & $\begin{array}{c}\text { Pooled Study (JPHC-I, JPHC-II, JACC, MIYAGI,3-pref } \\
\text { MIYAGI,3-pref AICHI) }\end{array}$ & Green tea & 219080 & 3577 & $40-103$ & $8-15$ & $\begin{array}{c}100479 \\
(45.9)\end{array}$ \\
\hline $\begin{array}{l}\text { Sauvaget et al. } \\
2005[52]\end{array}$ & Japan & Cohort & Atomic-Bomb Survivors (LSS Study) & Green tea & 38576 & 1270 & $34-98$ & 1980-1999 & $\begin{array}{l}14885 \\
(38.6)\end{array}$ \\
\hline $\begin{array}{l}\text { Galanis et al. } \\
1998 \text { [42] }\end{array}$ & Japan & Cohort & Population based & Green tea & 11907 & 108 & 46.4 & 14.8 & $\begin{array}{l}5610 \\
(47.1)\end{array}$ \\
\hline $\begin{array}{l}\text { Goldbohm } \\
\text { et al. } 1996 \text { [59] }\end{array}$ & Netherlands & Case-cohort & $\begin{array}{l}\text { Population based (Netherlands Cohort Study on Diet } \\
\text { and Cancer) }\end{array}$ & Black tea & 2929 & 182 & $55-69$ & 4.3 & $0(0)$ \\
\hline Reference & Response rate & Assessment of exposure & Adjustments & & & & & & $\begin{array}{c}\text { Quality } \\
\text { score } \\
\text { (NOS } \\
\text { stars) }\end{array}$ \\
\hline
\end{tabular}

\section{Breast Cancer}

Fagherazzi et al.

$2011[57]$

Iwasaki et al.

$2010[60]$

$>80 \%$

Dai et al.

$2010[33]$

2010 [56]
Self-administered FFQ

Total energy intake, ever use of oral contraceptives, age at menarche, age at menopause, number of children, age at first pregnancy, history of breast cancer in the family and years of schooling, current use of postmenopausal hormone therapy, personal history of benign breast disease, menopausal status and BMI

Age, area, age at menarche, menopausal status at baseline, number of births, age at first birth, height, BMl, alcohol intake, smoking status, leisure time physical activity, daily physical activity, exogenous hormone use, family history of breast cancer, oolong tea intake, black tea intake, coffee intake, canned coffee intake and Sencha and

Bancha/Genmaicha intake.

Age, educational achievement, income, family history of breast cancer, history of fibro adenoma, body mass index, (frequency of tea consumption) waist-to-hip ratio, physically active, smoking status, alcohol consumption status, passive smoking status, ginseng intake, age at menarche, age at first live birth, menopausal status, age at menopause, use of hormone replacement therapy, and dietary intake of total energy, fruits, vegetables, red meat, fish, and isoflavones.

Age, energy intake, age at menarche, BMI at age 18, family history of breast cancer, education, geographic region, parity, age at first birth, oral contraceptive use, menopausal status, age at menopause, female hormone use, vigorous activity, smoking status, alcohol intake, coffee and decaffeinated coffee

Propensity score (based on age, smoking status, educational status, BMl, alcohol intake, energy intake, energy adjusted saturated fat intake, energy adjusted fiber intake, coffee intake, physical activity level, ever use of oral contraceptives, presence of hypercholesterolemia, family history of breast cancer, age at menarche, parity, and cohort) 
$2009[61]$

Ishitani et al.

$2008[25]$
Self-administered FFO

Age, education, body mass index, height, parity, age at first birth, age at menarche, age at menopause, use of oral contraceptives, use of postmenopausal hormones, family history of breast cancer, intakes of total energy, alcohol and coffee

Age, randomized treatment assignment, body mass index, physical activity, total energy intake, alcohol intake, multivitamin use, age at menopause, age at menarche, age at first pregnancy lasting $\geq 6$ months, number of pregnancies lasting $\geq 6$ months, menopausal status, postmenopausal hormone use, prior hysterectomy, prior bilateral oophorectomy, smoking status, family history of breast cancer in mother or a sister, and history of benign breast disease

Age months, smoking status, body mass index, physical activity, height, alcohol intake, family history of breast cancer in mother or a sister, history of benign breast disease, menopausal status, age at menopause, use of hormone therapy, age at menarche, parity and age at first birth, weight change after18 and duration of postmenopausal hormone use and Coffee record

Self-administered FFQ

Age at menarche, parity, age at first birth, family history of breast cancer in mother and/or sister, history of benign breast disease, oral contraceptive use, alcohol consumption, energy intake, current body mass index, height, smoking habit, physical activity and menopausal status

Self-administered FFQ Age, types of health insurance, age at menarche, menopausal status, age at first birth, parity, mother's history of breast cancer, smoking, alcohol drinking, body mass index and consumption frequencies of black tea and coffee

Self-administered FFQ Age, family history of breast cancer, height, body mass index, education, parity, age at first birth, alcohol consumption, total caloric intake

Self-administered FFQ Attained age, calendar period, city, age at time of bombing and radiation dose

Self-administered FFQ

Age, education, smoking status, pack-years of smoking, physical activity, all fruit and vegetable Intake, waist/hip ratio, and family history of cancer, age at menarche, age at menopause, age at first pregnancy

Self-administered FFQ

Benign breast disease, history of breast cancer in mother and sisters, age at menarche, age at menopause, use of oral contraceptives, age atfirst birth, parity, body mass index, smoking status, education, and intakes of energy, fat, and alcohol

\section{Colorectal Cancer}

Self-administered FFQ Age, gender, race, family history of colorectal cancer, education, body mass index, physical activity, smoking status, NSAID intake, history of diabetes, number of colorectal examinations up to 3 years before the start of study, hormone use, fruit intake, vegetable intake, meat intake, alcohol intake and study centre.

Self-administered FFQ

Age, sex, race, education, smoking status, time since quitting for former smokers, smoking dose, ever smoke a pipe or cigar, diabetes, colorectal screening, family history of colorectal cancer, regular

non-steroidal anti-inflammatory drug use, marital status, BMl, frequency of vigorous physical activity, calories, fruit and vegetables, red meat, dietary calcium intake, alcohol, and menopausal hormone therapy in women

In-person interview (frequency of tea consumption)

Self-administered FFQ Age, education, cigarette smoking, pack-years of cigarette smoking, alcohol consumption, regular exercise, body mass index, history ofdiabetes, family history of colorectal cancer and intakes of vegetables, fruits and red meat ethanol intake, meat intake, processed meat intake, foliate intake, vitamin B6 intake, fiber intake, and fluid intake from other fluids

BMI, smoking status, alcohol drinking, family history of colorectal cancer, physical activity, and intake of green vegetables, beef, pork, green tea, Chinese tea and black tea 
Table 1 Main characteristics of the studies on tea consumption and five selected cancer included in the meta-analysis (Continued)

\begin{tabular}{|c|c|c|c|c|}
\hline $\begin{array}{l}\text { Oba et al. } \\
2006[31]\end{array}$ & $92 \%$ & Self-administered FFQ & $\begin{array}{l}\text { Age, height, BMl, total pack-years of cigarette smoking, alcohol intake, physical activity, black tea intake and green } \\
\text { tea/coffee intake. }\end{array}$ & 8 \\
\hline $\begin{array}{l}\text { Suzuki et al. } \\
2005[44]\end{array}$ & $91.7 \%$ & Self-administered FFQ & $\begin{array}{l}\text { Sex, age, family history of colorectal cancer, cigarette smoking, alcohol consumption, body mass index, consumption } \\
\text { of black tea, and coffee. Cohort1 adjusted for consumption of meat, green-yellow vegetables, other vegetables, } \\
\text { and fruits. Cohort2 adjusted for consumption of beef, pork, ham, chicken, liver, spinach, carrot or pumpkin, tomato, } \\
\text { orange, other fruits, and juice }\end{array}$ & 8 \\
\hline $\begin{array}{l}\text { Michels et al. } \\
2005 \text { [39] }\end{array}$ & $100 \%$ and $96 \%$ & Self-administered FFQ & $\begin{array}{l}\text { Age, family history of colorectal cancer, history of sigmoidoscopy, height, body mass index, pack-years of smoking, } \\
\text { physical activity, aspirin use, vitamin supplement intake, alcohol consumption, red meat consumption, total caloric } \\
\text { intake, and, among women in addition for menopausal status, postmenopausal hormone use. }\end{array}$ & 7 \\
\hline $\begin{array}{l}\text { Su et al. } \\
2002[28]\end{array}$ & $92.2 \%$ & $\begin{array}{l}\text { In-person interviews } \\
\text { (24 h food recall) }\end{array}$ & $\begin{array}{l}\text { Baseline age, race, education level, BMI, aspirin use, dietary intakes of calories, fat, fiber and calcium, and alcohol } \\
\text { use at baseline. }\end{array}$ & 9 \\
\hline $\begin{array}{l}\text { Terry et al. } \\
2001[45]\end{array}$ & $98 \%$ & Self-administered FFQ & $\begin{array}{l}\text { Age in 5-yr age groups, body mass index (quartiles), education level (3 categories), quartiles of total calories, red } \\
\text { meat, coffee, alcohol, energy-adjusted total fat, fruit fiber, vegetable fiber, cereal fiber, calcium, vitamin C, folic acid, } \\
\text { and vitamin D. }\end{array}$ & 8 \\
\hline $\begin{array}{l}\text { Nagano et al. } \\
2001[18]\end{array}$ & $72 \%$ & Self-administered FFQ & City, age, gender, radiation exposure, smoking status, alcohol drinking, body mass index, education level, calendar time & 6 \\
\hline $\begin{array}{l}\text { Hartman et al. } \\
1998[40]\end{array}$ & - & Self-administered FFQ & Age, intervention group, calcium, occupational physical activity, and BMI. & 7 \\
\hline $\begin{array}{l}\text { Zheng et al. } \\
1996[41]\end{array}$ & $42.3 \%$ & Self-administered FFQ & $\begin{array}{l}\text { Age, education, smoking status, pack-years of smoking, physical activity, all fruit and vegetable Intake, waist/hip } \\
\text { ratio, and family history of cancer }\end{array}$ & 7 \\
\hline $\begin{array}{l}\text { Goldbohm } \\
\text { et al. } 1996 \text { [59] }\end{array}$ & $96 \%$ & Self-administered FFQ & $\begin{array}{l}\text { Benign breast disease, history of breast cancer in mother and sisters, age at menarche, age at menopause, use } \\
\text { of oral contraceptives, age at first birth, parity, body mass index, smoking status, education, and intakes of energy, } \\
\text { fat, and alcohol }\end{array}$ & 8 \\
\hline \multirow[t]{2}{*}{$\begin{array}{l}\text { Nechuta et al. } \\
2012[27]\end{array}$} & $99.8 \%$ & $\begin{array}{l}\text { In-person interview, } \\
\text { self-administered FFQ }\end{array}$ & $\begin{array}{l}\text { age, marital status, education, occupation, BMI, exercise, fruit and vegetable intake, meat intake, diabetes, and } \\
\text { family history of digestive system cancer }\end{array}$ & 9 \\
\hline & & & Liver Cancer & \\
\hline $\begin{array}{l}\text { Nechuta et al. } \\
2012[27]\end{array}$ & $99.8 \%$ & $\begin{array}{l}\text { In-person interview, } \\
\text { self-administered FFQ }\end{array}$ & $\begin{array}{l}\text { age, marital status, education, occupation, BMI, exercise, fruit and vegetable intake, meat intake, diabetes, and } \\
\text { family history of digestive system cancer }\end{array}$ & 9 \\
\hline $\begin{array}{l}\text { Ui et al. } \\
2009[65]\end{array}$ & $94.6 \%$ & Self-administered FFQ & $\begin{array}{l}\text { Age, sex, alcohol consumption, smoking status, coffee consumption, vegetable consumption, dairy products } \\
\text { consumption, fruit consumption, fish consumption, soybean consumption }\end{array}$ & 8 \\
\hline $\begin{array}{l}\text { Inoue et al. } \\
2009 \text { [64] }\end{array}$ & $82 \%$ & Self-administered FFQ & $\begin{array}{l}\text { Sex, age, area, smoking status, weekly ethanol intake, body mass index, history of diabetes mellitus, coffee } \\
\text { consumption, green tea consumption, serum ALT level, HCV infection status, and HBV infection status }\end{array}$ & 8 \\
\hline \multirow[t]{2}{*}{$\begin{array}{l}\text { Nagano et al. } \\
2001[18]\end{array}$} & $72 \%$ & Self-administered FFQ & City, age, gender, radiation exposure, smoking status, alcohol drinking, body mass index, education level, calendar time & 7 \\
\hline & & & Prostate Cancer & \\
\hline $\begin{array}{l}\text { Geybels et al. } \\
2013 \text { [69] }\end{array}$ & $96 \%$ & Self-administered FFQ & Age & 8 \\
\hline $\begin{array}{l}\text { Montague et al. } \\
2012[30]\end{array}$ & UK & In-person Interview & Age, dialect group, interview year, education, body mass index and smoking history, green/black tea intake & 8 \\
\hline $\begin{array}{l}\text { Shafique et al. } \\
2012 \text { [68] }\end{array}$ & $70 \%$ & Self-administered FFQ & $\begin{array}{l}\text { Age, body mass index, smoking status, coffee consumption, alcohol intake, cholesterol level, systolic blood } \\
\text { pressure, social class, and years of full-time education }\end{array}$ & 7 \\
\hline
\end{tabular}


Table 1 Main characteristics of the studies on tea consumption and five selected cancer included in the meta-analysis (Continued)

\begin{tabular}{|c|c|c|c|c|}
\hline $\begin{array}{l}\text { Kurahashi et al. } \\
2008[67]\end{array}$ & $77 \%$ & Self-administered FFQ & $\begin{array}{l}\text { Age, area, smoking status, alcohol consumption, body mass index, marital status, and coffee, black tea, and } \\
\text { miso soup consumption, fruits, green or yellow vegetables, dairy food, soy food, and genistein consumption }\end{array}$ & 7 \\
\hline $\begin{array}{l}\text { Kikuchi et al. } \\
2006[66]\end{array}$ & $95 \%$ & Self-administered FFQ & $\begin{array}{l}\text { Age, body mass index, alcohol consumption, smoking status, marital status, daily calorie intake, daily calcium } \\
\text { intake, walking duration, consumption frequencies of black tea and coffee and consumption frequencies of fish }\end{array}$ & 8 \\
\hline $\begin{array}{l}\text { Allen et al. } \\
2004 \text { [50] }\end{array}$ & UK & Interview-based FFQ & Age, calendar period, city of residence, radiation dose and education level & 7 \\
\hline $\begin{array}{l}\text { Ellision et al. } \\
2000[53]\end{array}$ & $47 \%$ & $\begin{array}{l}\text { In-person interviews } \\
\text { ( } 24 \mathrm{~h} \text { food recall and one } \\
\text { month food frequency) }\end{array}$ & $\begin{array}{l}\text { Age, coffee, cola, total alcohol, beer, wine, spirits, smoking status, pack-years smoking, body mass index, highest } \\
\text { education level attained, respondent status, intake of fiber, fat, calories. }\end{array}$ & 8 \\
\hline \multicolumn{5}{|c|}{ Stomach Cancer } \\
\hline $\begin{array}{l}\text { Nechuta et al. } \\
2012[27]\end{array}$ & $99.8 \%$ & $\begin{array}{l}\text { In-person interview, } \\
\text { self-administered FFQ }\end{array}$ & $\begin{array}{l}\text { Age, marital status, education, occupation, BMl, exercise, fruit and vegetable intake, meat intake, diabetes, and } \\
\text { family history of digestive system cancer }\end{array}$ & 9 \\
\hline $\begin{array}{l}\text { Inoue et al. } \\
2009[43]\end{array}$ & $\begin{array}{l}82 \%, 80 \%, 83 \% \\
92 \%, 94 \%, 90 \%\end{array}$ & Self-administered FFQ & $\begin{array}{l}\text { Age, area, smoking, ethanol intake, rice intake, soy bean paste soup, and coffee intake, pickled vegetable intake } \\
\text { and green-yellow vegetable intake }\end{array}$ & 8 \\
\hline $\begin{array}{l}\text { Sauvaget et al. } \\
2005[52]\end{array}$ & $72.5 \%$ & Self-administered FFQ & Sex, sex-specific age, city, radiation dose, sex-specific smoking habits, and education level. & 6 \\
\hline $\begin{array}{l}\text { Galanis et al. } \\
1998[42]\end{array}$ & $95 \%$ & Self-administered FFQ & $\begin{array}{l}\text { Age, years of education, Japanese place of birth, and gender. Analyses among men were also adjusted for } \\
\text { cigarette smoking and alcohol intake status }\end{array}$ & 8 \\
\hline $\begin{array}{l}\text { Goldbohm } \\
\text { et al. } 1996[59]\end{array}$ & $72 \%$ & Self-administered FFQ & $\begin{array}{l}\text { Benign breast disease, history of breast cancer in mother and sisters, age at menarche, age at menopause, use of } \\
\text { oral contraceptives, age at first birth, parity, body mass index, smoking status, education, and intakes of energy, } \\
\text { fat, and alcohol }\end{array}$ & 7 \\
\hline
\end{tabular}




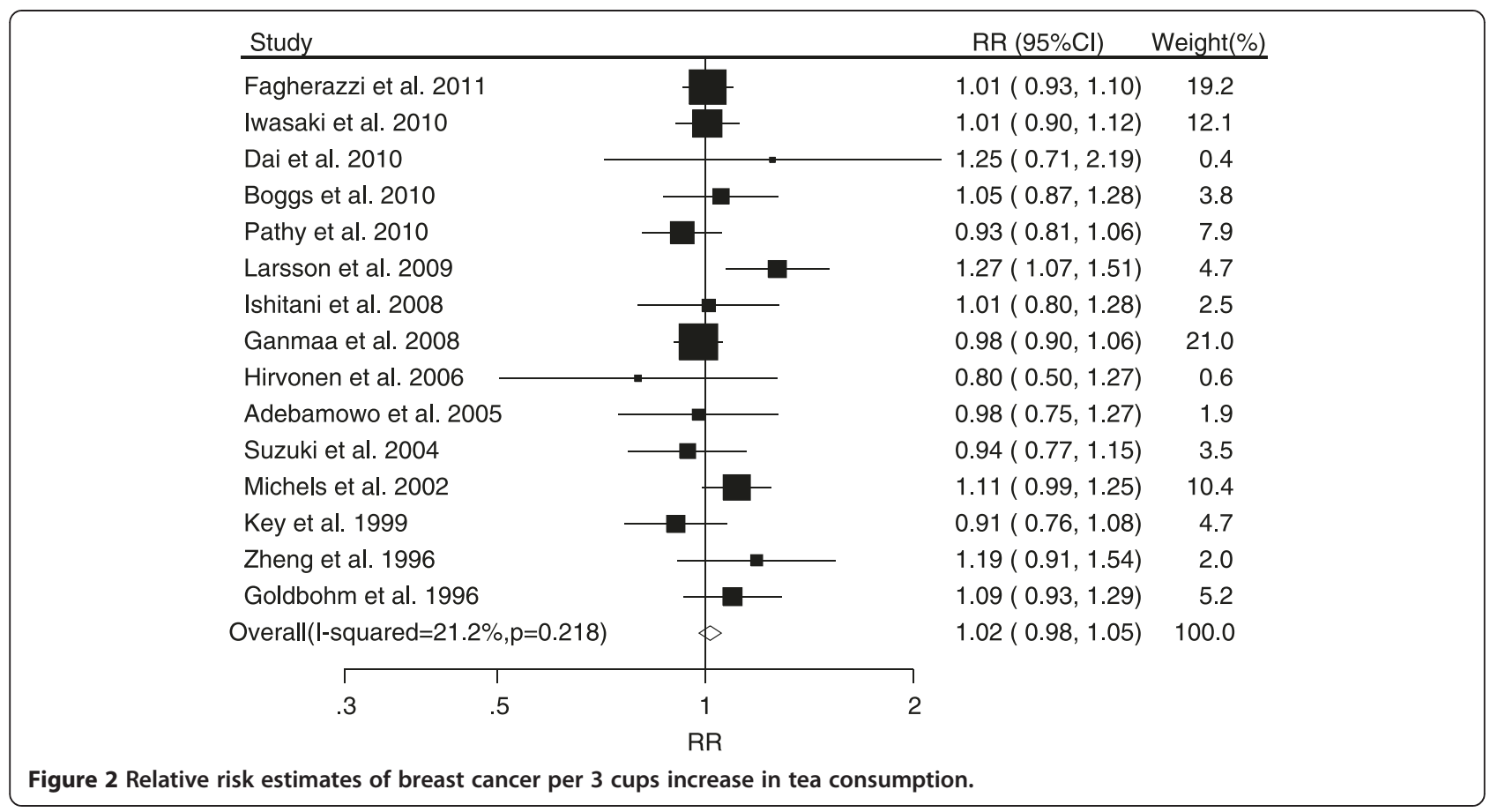

regions, and type of tea, showed no significant associations with risk of cancer.

The results of heterogeneity test suggested that most pooled effects had mild heterogeneity. However, moderate heterogeneity was seen in studies on liver cancer. Sensitivity analysis performed by omitting one study at a time, identified the main source of heterogeneity as the study by Inoue et al. [64], which included hepatitis C and $B$ virus positive patients while the other three studies did not. After excluding this study, the overall RR was $0.86(95 \% \mathrm{CI}, 0.75-0.99)$ with a mild heterogeneity $\left(P=0.30, I^{2}=17.1 \%\right)$, which suggests a preventive effect of tea drinking for liver cancer. However, large prospective studies are still needed to verify this preventive effect.

Eleven previous meta-analyses have evaluated the association between green tea or black tea intake and the risk of the five selected cancers [12-16,70-75]. Of these earlier meta-analyses, three focused on breast cancer, three on colorectal cancer, three on stomach cancer, one on primary liver cancer, and one on prostate cancer. All of the previous eleven meta-analyses reported the summarized RR/OR for the highest tea consumption level relative to the no tea/lowest level. In nine of the metaanalyses that examine the association between green tea consumption and cancer risk, three studies presented an overall statistically significant inverse association between green tea consumption and cancer risk when they included both cohort and case-control studies in the meta-analysis. The summarized ORs of these three studies were 0.82 (95\% CI, 0.70-0.96) and 0.86 (95\% CI, 0.74-
1.00) for stomach cancer and 0.82 (95\% CI, $0.69-0.98)$ for colorectal cancer $[71,72,75]$. Among the eleven metaanalyses, seven studies reported an inverse association between green tea consumption and cancer risk from the summarized results of case-control studies. However, none of the studies indicated a statistically significant green tea-cancer risk association from a metaanalysis of prospective cohort studies only. These earlier results from prospective studies were consistent with our present meta-analysis. For a concise comparison, we have presented the pooled RRs of previous studies and our study in Table 3 .

Among the three meta-analyses examing the relationship between black tea consumption and cancer risk, Sun et al. presented conflicting results across cohort studies and case-control studies for breast cancer risk [73]. The pooled RR for prospective cohort studies was 1.15 (95\% CI, 1.02 to 1.31), while the pooled RR for case-control studies was 0.91 (95\% CI, 0.84 to 0.98 ). Our meta-analysis also found a positive association between black tea consumption and the risk of breast cancer in a subgroup analysis. The summarized RR was 1.18 (95\% CI, 1.05-1.32) with an increment of three cups black tea intake per day. Association of black tea consumption with breast cancer risk is biologically plausible. Black tea intake has been positively associated the estrogen levels, and experimental studies have established that estrogen is a strong promoter of mammary carcinogenesis [76]. Wu et al. reported that the levels of circulating estrogens were higher in black tea drinkers than in non-tea drinkers [77], and Larsson et al. reported that 


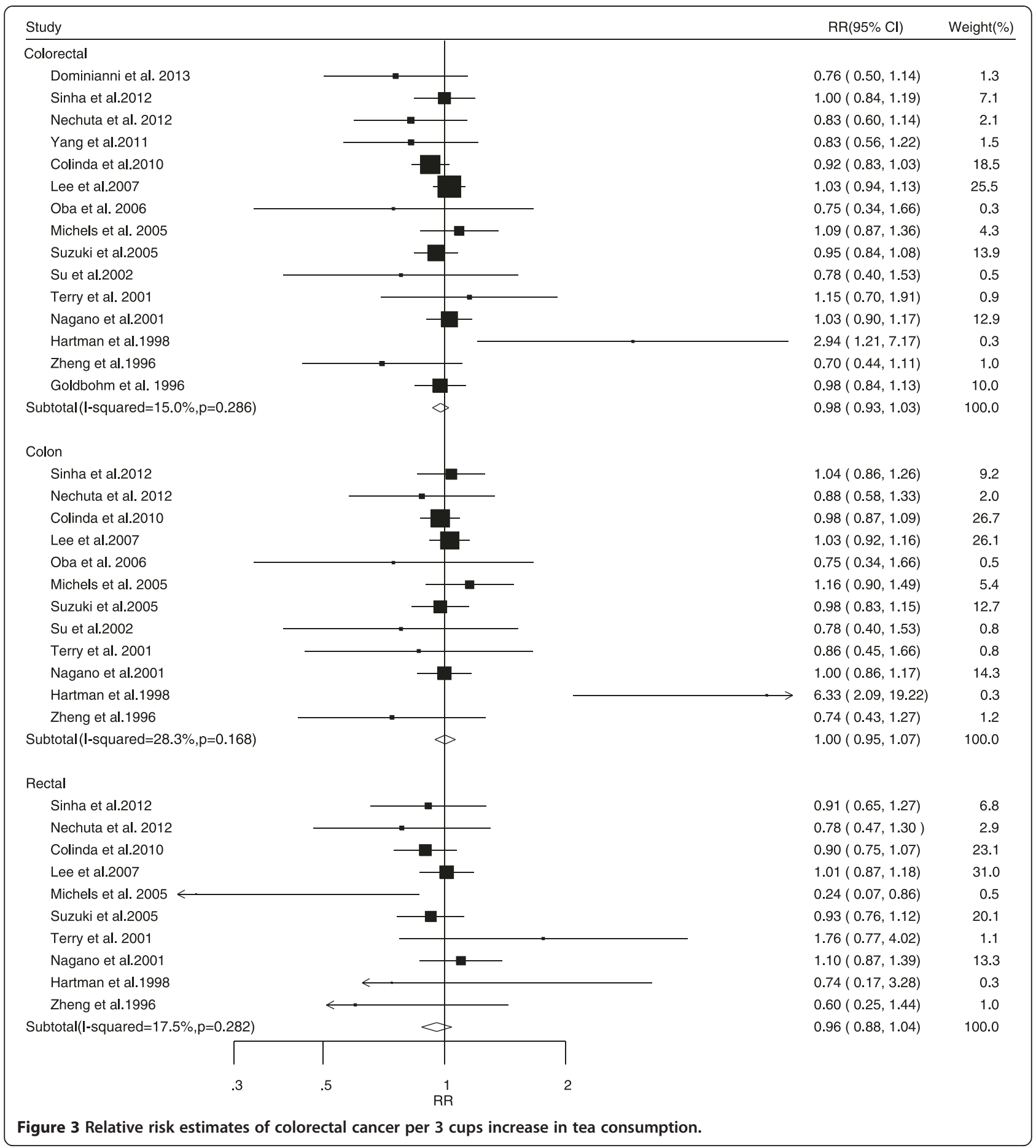

black tea consumption was positively associated with the risk of $E R+/ P R+$ breast cancer, suggesting a possible carcinogenic role involving sex hormones [61].

Of the three meta-analyses exploring the association between tea consumption and colorectal cancer risk, Zhang et al. [74] found a positive association between tea and colorectal cancer (RR 1.28; 95\% CI, 1.02-1.61) for tea consumption greater than four cups. Our pooled
RR was 0.98 (95\% CI, 0.93-1.03) with an increment of three cups black tea intake per day. These differing results could be attributed to the calculation using different increment cups per day. We concluded that drinking three cups more per day does not increase the risk of colorectal cancer while Zhang et al. concluded that drinking four cups increment per day might be a risk factor for colon cancer. In addition, Zhang et al. also 


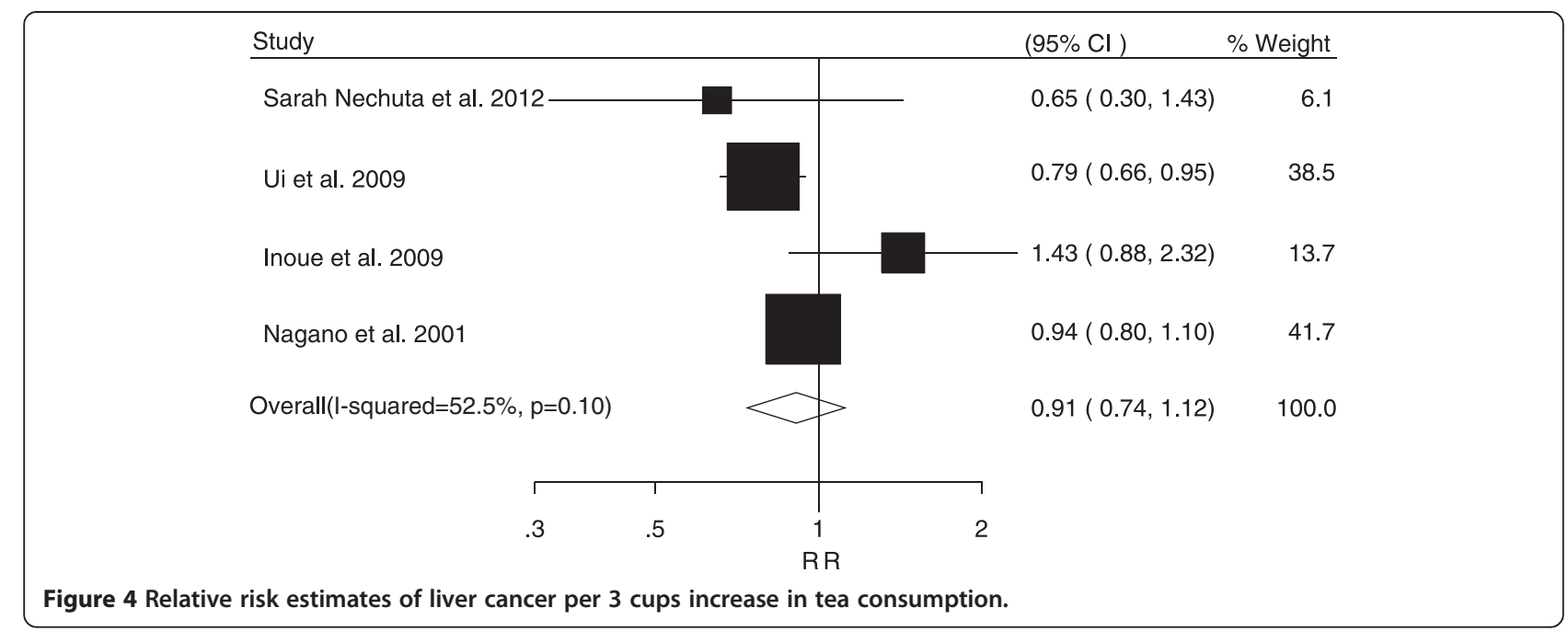

reported that the risk of colon cancer did not increase with a $250 \mathrm{~g} /$ day increment of tea consumption either in men (RR, 1.03; 95\% CI, 0.96-1.09) or women (RR, 1.03; 95\% CI, 0.99-1.08); this is consistent with our current meta-analysis. Zhang et al. attributed the observed positive association to chance.

It is notable that most epidemiological evidence for the inverse association between tea intake and cancer risk has come from retrospective case-control studies. Because information in case-control studies is collected after cancer is diagnosed, it reflects the past exposure history based on recall; therefore, recall bias is inevitable and cannot be ignored. This bias may partly explain the difference in the findings between prospective studies and retrospective case-control studies. Given the limitations of case-control studies, the conclusion that tea consumption has an inverse association with cancer risk is not convincing.

When compared with previous meta-analyses, our meta-analysis has several strengths. First, we assessed the association of tea consumption with five major cancers. Second, only prospective studies were included in the meta-analysis, which greatly reduces the likelihood of selection and recall biases. Third, the dose-response analysis included a wide range of tea consumption, which allows a concrete and quantitative assessment of the dose-response relationship between tea intake and cancer risk.

However, several potential limitations of our metaanalysis must be considered when interpreting the results. First, the temperature of tea is an important confounding factor when assessing the association between

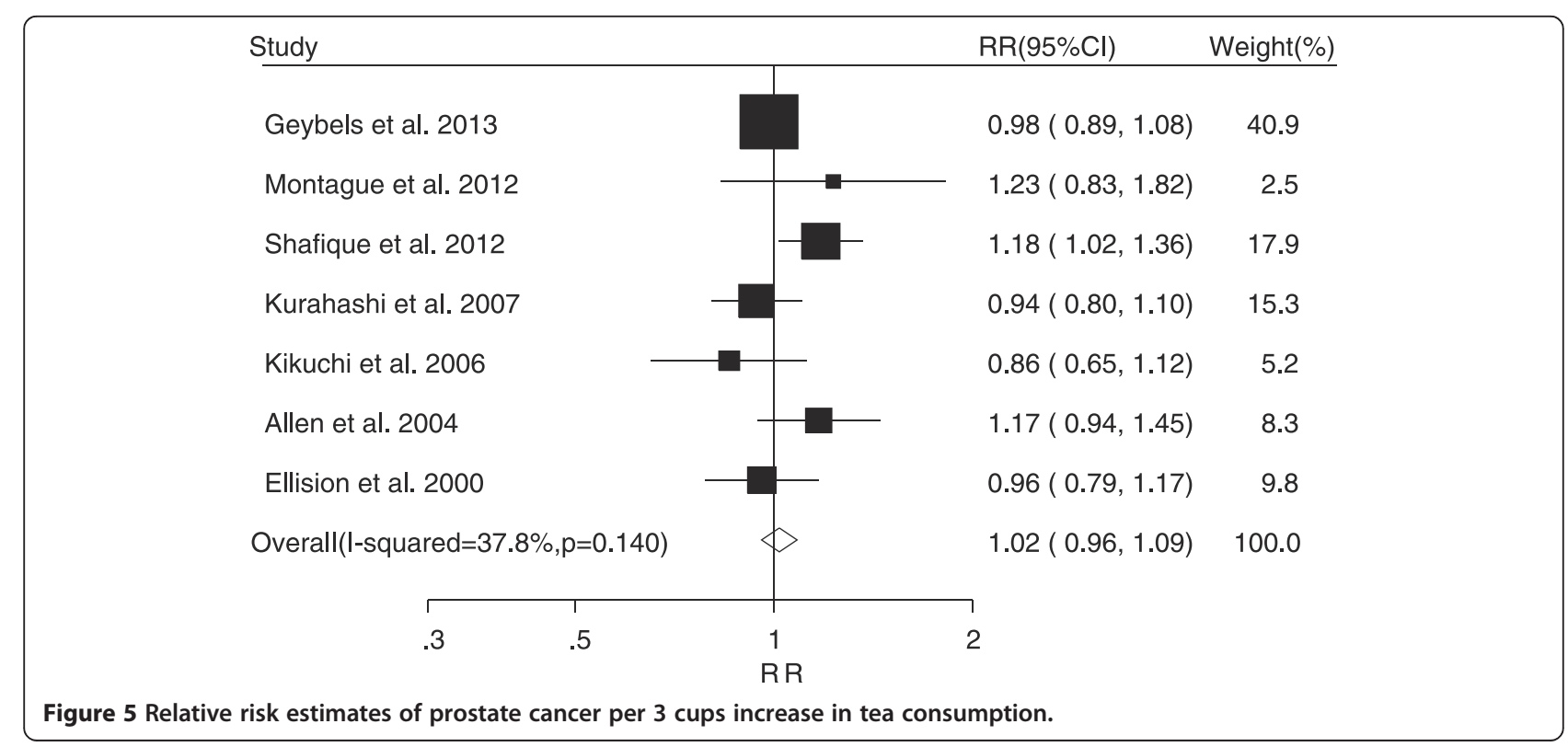




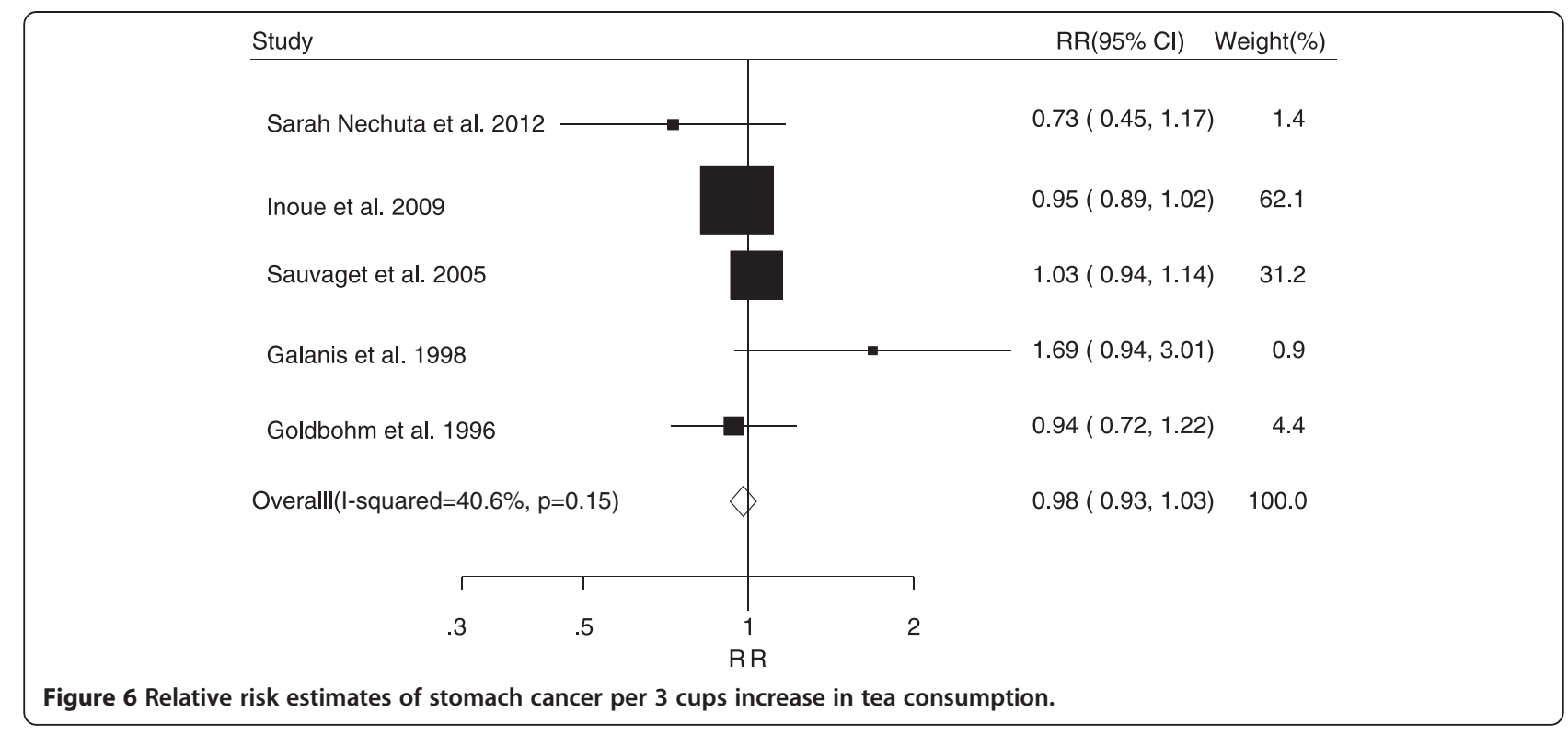

tea drinking and cancer risk. However, we could not analyze the effect of this factor because only one study reported the relationship between iced or hot tea and cancer; this study reported no significant association were observed between iced or hot tea and gastric cancers. Second, the methods of data collection differed across the included studies. Most of the studies assessed the exposure of the tea consumption via food frequency questionnaire, and the response rate varied among these studies. As is known, people in different countries have different traditions of tea consumption or drinking style, which presents difficulties in assessing tea consumption amounts accurately. In addition, people from the same country may also have different habits of drinking tea, such as strong or weak tea. In addition, tea consumption level is mostly assessed as the number of cups of tea consumed daily or weekly. However, cup size may vary considerably for different countries or areas and the dry tea leaves brewed in each cup may also be different. The cut-offs for the highest consumption level varied across different studies. Therefore, there might be some inevitable measurement errors and possible uncontrolled confounding factors when assessing tea consumption; this could prevent the detection of a modest association between tea consumption and cancer risk. However, we endeavoured to decrease these errors by unifying the unit of measurement and using a similar standard to calculate daily tea consumption. We also performed sensitivity analyses by removing the studies that did not report tea consumption as cups and performed subgroup analyses by geographic region, especially China and Japan (see Table 2). Third, we had no information about the family history of certain cancers in the primary aggregate results. Genetic factors play an important role in the development of cancers. Some genetic factors may increase the susceptibility to cancer, and certain polymorphisms in genes that are responsible for metabolising tea may have a role in the tea-cancer association. Fourth, a meta-analysis is unable to account for confounding factors inherent in the original studies. Although major potential confounders, including age, sex, alcohol, and smoking had been adjusted in most included studies, residual or unknown confounding cannot be excluded as a potential explanation for the observed findings. It is known that alcohol and smoking are important potential confounding factors. For example, people in China who drink tea frequently are more likely to drink more alcohol or smoke. The interplay of these factors, tea, alcohol, and smoking, could not be detected because of limited data. Most of the included studies did not provide the numbers for case and non-case population at each level of tea consumption; therefore, based on the available data, analyses among non-smokers and non-alcohol drinkers could not be conducted. Additionally, not all studies adjusted for these confounding factors. For breast cancer, the menopausal status of women was an important confounding factor. However, according to our subgroup analysis on pre-menopausal (RR, 0.96; 95\% CI, 0.79-1.18) and post-menopausal (RR, 1.12; $95 \% \mathrm{CI}, 0.96-1.30$ ) women, there were no significant risk associations between tea consumption and pre-menopausal or post-menopausal breast cancer. Considering only three studies were available for this subgroup analysis, the results may be because of limited sample size. Thus, menopausal status could be another effect modifier for breast cancer, and further studies are needed to discuss the confounding effect of this factor for breast cancer [33]. Finally, publication bias is an 


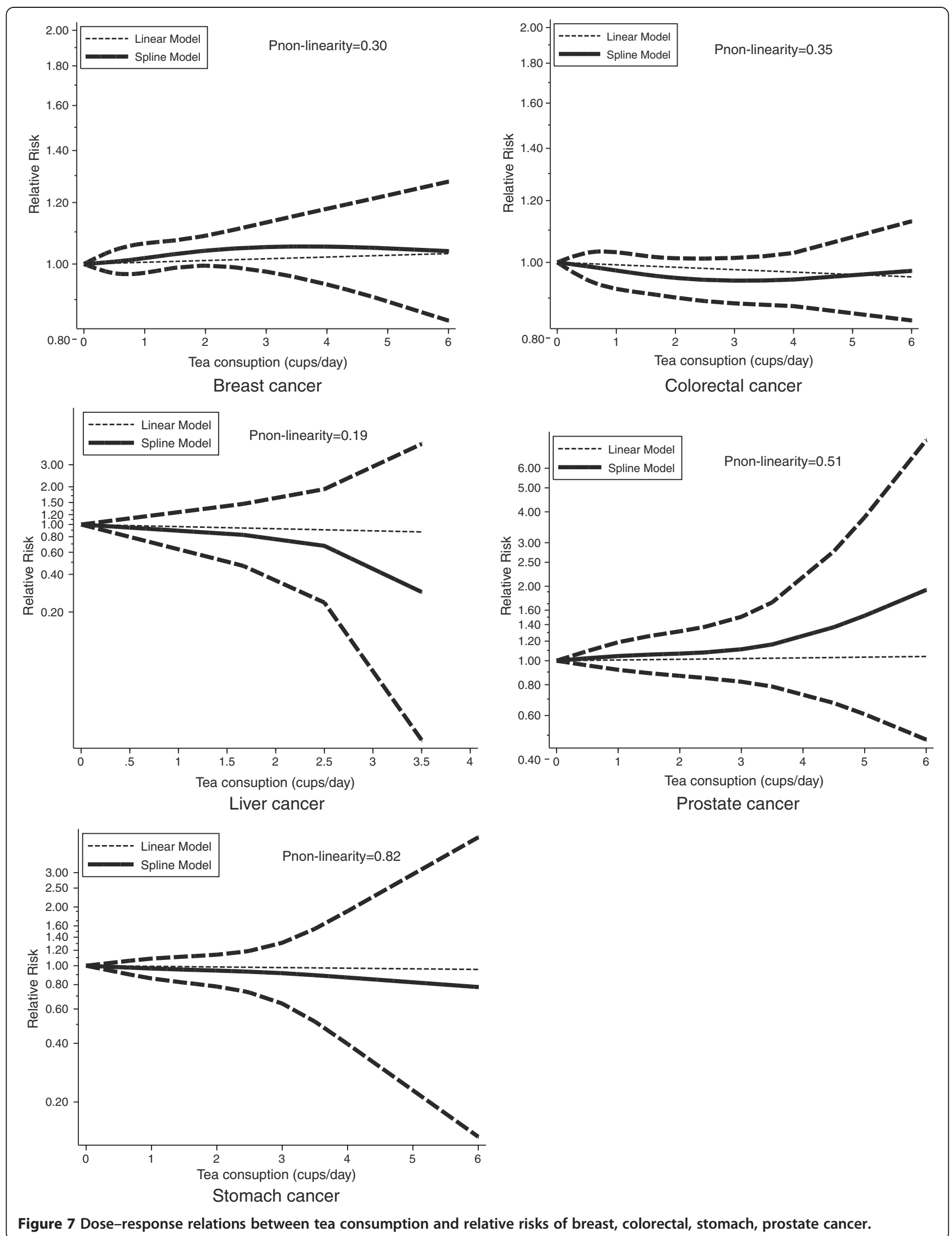


Table 2 Subgroup analysis of cancer risk for an increment of three cup tea consumption by gender, tea type and geographic region

\begin{tabular}{|c|c|c|c|c|c|c|c|c|}
\hline \multirow[t]{2}{*}{ Subgroup } & \multicolumn{2}{|r|}{ Breast } & \multicolumn{2}{|r|}{ Stomach } & \multicolumn{2}{|r|}{ Liver } & \multicolumn{2}{|r|}{ Prostate } \\
\hline & $N$ & Pooled RR $(95 \% \mathrm{Cl})$ & $N$ & Pooled RR $(95 \% \mathrm{Cl})$ & $N$ & Pooled RR $(95 \% \mathrm{Cl})$ & $N$ & Pooled RR $(95 \% \mathrm{Cl})$ \\
\hline \multicolumn{9}{|l|}{ By gender } \\
\hline Male & - & - & 2 & $1.20(0.72-2.01)$ & - & - & 6 & $1.02(0.96-1.09)$ \\
\hline Female & 15 & $1.02(0.98-1.05)$ & 3 & $0.88(0.80-0.98)$ & 1 & $0.65(1.30-1.43)$ & - & - \\
\hline \multicolumn{9}{|c|}{ By menopausal status } \\
\hline Pre-menopausal & 2 & $0.96(0.79-1.18)$ & - & & - & & - & \\
\hline Post-menopausal & 3 & $1.12(0.96-1.30)$ & - & & - & & - & \\
\hline \multicolumn{9}{|l|}{ By tea type } \\
\hline Green tea & 4 & $0.97(0.90-1.06)$ & 4 & $0.99(0.94-1.05)$ & 3 & $0.93(0.75-1.17)$ & 4 & $0.99(0.88-1.11)$ \\
\hline Black tea & 4 & $1.18(1.05-1.32)$ & 1 & $0.94(0.72-1.22)$ & - & - & 3 & $0.99(0.90-1.09)$ \\
\hline \multicolumn{9}{|c|}{ By geographic region } \\
\hline Europe & 6 & $1.05(0.96-1.15)$ & 1 & $0.94(0.72-1.22)$ & - & - & 2 & $1.07(0.90-1.27)$ \\
\hline Asian & 4 & $0.98(0.90-1.06)$ & 4 & $0.99(0.87-1.12)$ & 4 & $0.91(0.74-1.12)$ & 4 & $1.00(0.90-1.12)$ \\
\hline China & 1 & $1.25(0.71-2.19)$ & 1 & $0.73(0.45-1.17)$ & 1 & $0.65(0.30-1.43)$ & - & - \\
\hline Japan & 3 & $0.97(0.89-1.05)$ & 3 & $1.01(0.86-1.14)$ & 3 & $0.90(0.80-1.01)$ & 3 & $0.99(0.88-1.11)$ \\
\hline North America & 5 & $1.00(0.94-1.07)$ & - & - & - & - & 1 & $0.96(0.79-1.17)$ \\
\hline \multirow[t]{2}{*}{ Subgroup } & & & \multicolumn{2}{|r|}{ Colon } & \multicolumn{2}{|r|}{ Rectal } & \multirow[b]{2}{*}{$N$} & \multirow{2}{*}{$\begin{array}{c}\text { Colorectal } \\
\text { Pooled RR }(95 \% \mathrm{Cl}\end{array}$} \\
\hline & & & $N$ & Pooled RR $(95 \% \mathrm{Cl})$ & $N$ & Pooled RR $(95 \% \mathrm{Cl})$ & & \\
\hline \multicolumn{9}{|l|}{ By gender } \\
\hline Male & & & 6 & $1.00(0.90-1.11)$ & 5 & $0.96(0.83-1.11)$ & 6 & $0.98(0.90-1.07)$ \\
\hline Female & & & 6 & $1.02(0.92-1.14)$ & 2 & $0.93(0.78-1.10)$ & 6 & $0.99(0.90-1.09)$ \\
\hline \multicolumn{9}{|l|}{ By tea type } \\
\hline Green tea & & & 4 & 1.01 (0.93-1.09) & 3 & $1.00(0.90-1.12)$ & 5 & $1.00(0.94-1.07)$ \\
\hline Black tea & & & 1 & $0.86(0.45-1.66)$ & 1 & $1.76(0.77-4.02)$ & 2 & $0.99(0.86-1.14)$ \\
\hline \multicolumn{9}{|c|}{ By geographic region } \\
\hline Europe & & & 3 & $1.02(0.84-1.24)$ & 3 & $0.92(0.77-1.10)$ & 4 & $0.96(0.88-1.04)$ \\
\hline Asian & & & 5 & $1.00(0.93-1.08)$ & 4 & $0.99(0.89-1.10)$ & 6 & $0.99(0.93-1.06)$ \\
\hline China & & & 1 & $0.88(0.58-1.33)$ & 1 & $0.78(0.47-1.30)$ & 2 & $0.83(0.64-1.06)$ \\
\hline Japan & & & 4 & 1.01 (0.93-1.09) & 3 & $1.00(0.90-1.12)$ & 4 & $1.01(0.94-1.07)$ \\
\hline North America & & & 4 & $1.04(0.90-1.20)$ & 3 & $0.81(0.60-1.09)$ & 5 & $0.97(0.85-1.09)$ \\
\hline
\end{tabular}

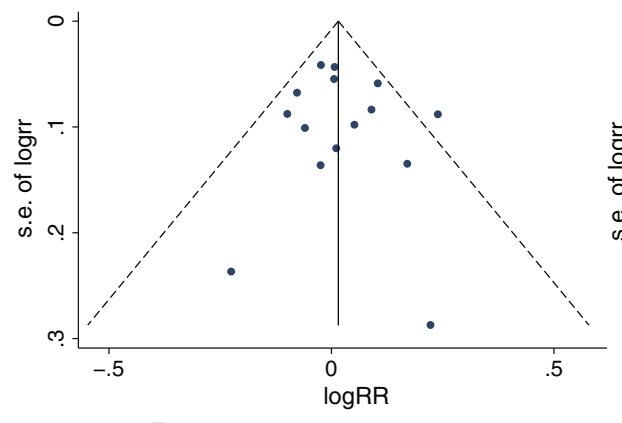

Tea consumption and breast cancer Egger test: $p=0.59 \quad$ Begg test $p=0.60$

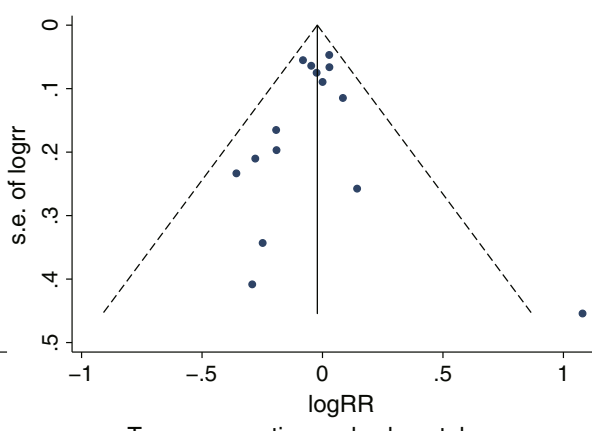

Tea consumption and colorectal cancer Egger test: $p=0.59$ Begg test: $p=0.73$

Figure 8 Funnel plot of log relative risk vs standard error of log relative risks. 
Table 3 omparison of findings of the present dose-response meta-analysis with those reported in previous meta-analysis

\begin{tabular}{|c|c|c|c|c|c|c|c|c|c|c|}
\hline \multirow[t]{3}{*}{ Reference } & \multirow[t]{3}{*}{ Tea type } & \multicolumn{6}{|c|}{ Previous review* } & \multicolumn{3}{|c|}{ Present review ${ }^{\#}$} \\
\hline & & \multicolumn{2}{|c|}{ Cohort study } & \multicolumn{2}{|c|}{ Case-control study } & \multicolumn{2}{|r|}{ Over all } & \multirow[b]{2}{*}{ Tea type } & \multirow[b]{2}{*}{$N$} & \multirow[b]{2}{*}{ Pooled RR $(95 \% \mathrm{Cl})$} \\
\hline & & $N$ & Pooled RR (95\% Cl) & $N$ & Pooled OR (95\% Cl) & $N$ & Pooled RR (95\% Cl) & & & \\
\hline \multicolumn{11}{|c|}{ Breast cancer } \\
\hline Ogunleye et al. 2010 & Green tea & 2 & $0.85(0.65-1.12)$ & 5 & $0.81(0.75-0.88)$ & 7 & $0.81(0.75-0.88)$ & Tea & 15 & $1.02(0.98-1.06)$ \\
\hline Sun et al. 2006 & Green tea & 3 & $0.85(0.66-1.09)$ & 1 & $0.47(0.26-0.85)$ & 4 & $0.78(0.61-0.98)$ & Green tea & 4 & $0.97(0.90-1.06)$ \\
\hline Sun et al. 2006 & Black tea & 5 & $1.15(1.02-1.31)$ & 8 & $0.91(0.84-0.98)$ & 13 & $0.98(0.88-1.09)$ & Black tea & 4 & $1.18(1.05-1.32)$ \\
\hline Seely et al. 2005 & Green tea & 3 & $0.89(0.71-1.10)$ & 2 & $0.44(0.14-1.31)$ & - & - & & & \\
\hline \multicolumn{11}{|c|}{ Colorectal cancer } \\
\hline Wang et al. 2012 & Green tea & - & - & 13 & $0.95(0.81-1.11)$ & - & - & Tea & 15 & $0.98(0.93-1.03)$ \\
\hline Sun et al. 2006 & Green tea & 4 & $0.97(0.82-1.16)$ & 4 & $0.74(0.63-0.86)$ & 8 & $0.82(0.69-0.98)$ & Green tea & 5 & $1.00(0.94-1.07)$ \\
\hline Sun et al. 2006 & Black tea & 7 & $1.02(0.78-1.34)$ & 13 & $0.98(0.84-1.15)$ & 20 & $0.99(0.87-1.13)$ & Black tea & 2 & $0.99(0.86-1.14)$ \\
\hline Zhang et al. 2010 & Tea & 13 & $1.28(1.02-1.61)$ & - & - & 13 & $1.28(1.02-1.61)$ & & & \\
\hline \multicolumn{11}{|c|}{ Liver cancer } \\
\hline \multirow[t]{2}{*}{ Sing et al. 2011} & Tea & 7 & $0.84(0.69-1.02)$ & 6 & $0.86(0.44-1.14)$ & 11 & $0.77(0.57-1.03)$ & Tea Green tea & 4 & $0.93(0.75-1.17)$ \\
\hline & & & & & & & & & 3 & $0.93(0.75-1.17)$ \\
\hline \multicolumn{11}{|c|}{ Prostate cancer } \\
\hline Zheng et al. 2011 & Green tea & 4 & $1.00(0.66-1.53)$ & 3 & $0.43(0.25-0.73)$ & 7 & $0.72(0.45-1.15)$ & Tea & 7 & $1.00(0.87-1.15)$ \\
\hline \multirow[t]{2}{*}{ Zheng et al. 2011} & Black tea & Prospective: 3 & $0.83(0.63-1.08)$ & 6 & $1.07(0.78-1.48)$ & 11 & $0.99(0.82-1.20)$ & Green tea & 4 & $0.99(0.88-1.11)$ \\
\hline & & Retrospective: 2 & $1.04(0.73-1.50)$ & & & & & Black tea & 3 & $0.99(0.90-1.09)$ \\
\hline \multicolumn{11}{|c|}{ Stomach cancer } \\
\hline Kang et al. 2010 & Green tea & 7 & $1.03(0.92-1.16)$ & 11 & $0.74(0.63-0.86)$ & 18 & $0.86(0.74-1.00)$ & Tea & 5 & $0.97(0.92-1.02)$ \\
\hline Myung et al. 2009 & Green tea & 7 & $1.04(0.93-1.17)$ & 8 & $0.73(0.64-0.83)$ & 15 & $0.82(0.70-0.96)$ & Green tea & 4 & $0.99(0.94-1.05)$ \\
\hline \multirow[t]{2}{*}{ Zhou et al. 2008} & Green tea & 4 & $1.56(0.93-2.60)$ & HCC:4 & $1.12(0.70-1.77)$ & 14 & $0.98(0.77-1.24)$ & Black tea & 1 & $0.94(0.72-1.22)$ \\
\hline & & & & PCC:6 & $0.67(0.49-0.92)$ & & & & & \\
\hline
\end{tabular}

HCC: Hospital based case-control PCC: population based case-control $N$ : number of studies included.

*Pooled results between highest consumption level and non/lowest drinker.

"Pooled results with an increase in tea consumption. 
inevitable problem in systematic reviews and metaanalyses. However, the results of publication bias analysis showed that there was no significant evidence of publication bias in studies on breast and colorectal cancer. The limited number of included studies precludes us from conducting publication bias analyses for liver, prostate, or stomach cancer.

\section{Conclusion}

There is insufficient information from epidemiologic studies to support the suggestion that tea intake plays a role in the prevention of cancer. Randomized controlled trials and large prospective cohort studies are needed to further explore this association.

\section{Additional file}

Additional file 1: The MOOSE checklist for this meta-analysis.

\section{Abbreviations}

GTE: Green tea extract; RR: relative risk; 95\% Cl: 95\% confidence intervals; HR: Hazard ratio; OR: Odds ratio; NOS: Newcastle-Ottawa Scale.

\section{Competing interests}

The author's declare that they have no competing interest.

\section{Author's contributions}

FY developed inclusion and exclusion criteria, drafted and revised the manuscript, contributed to the study design, analysis, and interpretation of the data. ZJ drafted the manuscript and contributed to the study design. $\mathrm{H}$ J contributed to revising the manuscript, data analysis and interpretation of the data. CX developed inclusion and exclusion criteria, contributed to the data analysis and interpretation of the data. JT and TL contributed to the electronic search, hand-search of literature and the revision of the manuscript. $\mathrm{JH}$ contributed to the interpretation of the results and the conception of the study. All authors read and approved the final manuscript.

\section{Acknowledgement}

This work was supported by two National Natural Science Foundation (81001287 and 81373105), three Ministry of Science and Technology of China (2008ZX10002-007, 2008ZX10002-018, and 2008ZX10002-025), a grant from the Leading Talents of Science in Shanghai 2010 (022) and the key discipline construction of evidence-based public health in Shanghai (12GWZX0602).

\section{Author details}

${ }^{1}$ Department of Health Statistics, Second Military Medical University, 800 Xiangyin Road, Shanghai 200433, China. ${ }^{2}$ Office of compliance and Development, Center for drug evaluation, China food and drug administration, Shanghai, China. ${ }^{3}$ Department of Endocrinology, Changzheng Hospital, Second Military Medical University, Shanghai 200003, China.

Received: 5 November 2013 Accepted: 11 March 2014 Published: 17 March 2014

\section{References}

1. Food and Agriculture Organization of the United Nations: Food balance sheets, 1994-1996. Available from: http://faostat3.fao.org/home/index. htmI\#DOWNLOAD_STANDARD.

2. Shimizu M, Adachi S, Masuda M, Kozawa O, Moriwaki H: Cancer chemoprevention with green tea catechins by targeting receptor tyrosine kinases. Mol Nutr Food Res 2011, 55(6):832-843.

3. Suzuki Y, Miyoshi N, Isemura M: Health-promoting effects of green tea. Proc Jpn Acad Ser B Phys Biol Sci 2012, 88(3):88-101.
4. Ahmad N, Mukhtar H: Green tea polyphenols and cancer: biologic mechanisms and practical implications. Nutr Rev 1999, 57(3):78-83.

5. Lambert JD: Does tea prevent cancer? Evidence from laboratory and human intervention studies. Am J Clin Nutr 2013, 98(6):1667S-1675S.

6. World Cancer Research Fund/American Institute for Cancer Research: Food, Nutrition, Physical Activity, and the Prevention of Cancer: a Global Perspective. Washington DC: AICR; 2007.

7. Choan E, Segal R, Jonker D, Malone S, Reaume N, Eapen L, Gallant V: A prospective clinical trial of green tea for hormone refractory prostate cancer: an evaluation of the complementary/alternative therapy approach. Urol Oncol 2005, 23(2):108-113.

8. Nguyen MM, Ahmann FR, Nagle RB, Hsu CH, Tangrea JA, Parnes HL, Sokoloff $\mathrm{MH}$, Gretzer MB, Chow HH: Randomized, double-blind, placebo-controlled trial of polyphenon $\mathrm{E}$ in prostate cancer patients before prostatectomy: evaluation of potential chemopreventive activities. Cancer Prev Res (Phila) 2012, 5(2):290-298.

9. Tsao AS, Liu D, Martin J, Tang XM, Lee JJ, El-Naggar AK, Wistuba I, Culotta KS, Mao L, Gillenwater A, Sagesaka YM, Hong WK, Papadimitrakopoulou V: Phase II randomized, placebo-controlled trial of green tea extract in patients with high-risk oral premalignant lesions. Cancer Prev Res (Phila) 2009, 2(11):931-941.

10. Sinha R, Cross AJ, Daniel CR, Graubard BI, Wu JW, Hollenbeck AR, Gunter MJ, Park $Y$, Freedman ND: Caffeinated and decaffeinated coffee and tea intakes and risk of colorectal cancer in a large prospective study. Am J Clin Nutr 2012, 96(2):374-381.

11. Yang G, Zheng W, Xiang YB, Gao J, Li HL, Zhang X, Gao YT, Shu XO: Green tea consumption and colorectal cancer risk: a report from the Shanghai Men's Health Study. Carcinogenesis 2011, 32(11):1684-1688.

12. Ogunleye AA, Xue F, Michels KB: Green tea consumption and breast cancer risk or recurrence: a meta-analysis. Breast Cancer Res Treat 2010, 119(2):477-484.

13. Sing MF, Yang WS, Gao S, Gao J, Xiang YB: Epidemiological studies of the association between tea drinking and primary liver cancer: a metaanalysis. Eur J Cancer Prev 2011, 20(3):157-165

14. Sun CL, Yuan JM, Koh WP, Yu MC: Green tea, black tea and colorectal cancer risk: a meta-analysis of epidemiologic studies. Carcinogenesis 2006, 27(7):1301-1309.

15. Wang XJ, Zeng XT, Duan XL, Zeng HC, Shen R, Zhou P: Association Between Green Tea and Colorectal Cancer Risk: A Meta-analysis of 13 Case-control Studies. Asian Pac J Cancer Prev 2012, 13(7):3123-3127.

16. Zheng J, Yang B, Huang T, Yu Y, Yang J, Li D: Green tea and black tea consumption and prostate cancer risk: an exploratory meta-analysis of observational studies. Nutr Cancer 2011, 63(5):663-672.

17. Mao Q, Lin Y, Zheng X, Qin J, Yang K, Xie L: A meta-analysis of alcohol intake and risk of bladder cancer. Cancer Causes Control 2010, 21(11):1843-1850.

18. Nagano J, Kono S, Preston DL, Mabuchi K: A prospective study of green tea consumption and cancer incidence, Hiroshima and Nagasaki (Japan). Cancer Causes Control 2001, 12(6):501-508.

19. Ren JS, Freedman ND, Kamangar F, Dawsey SM, Hollenbeck AR, Schatzkin A, Abnet CC: Tea, coffee, carbonated soft drinks and upper gastrointestinal tract cancer risk in a large United States prospective cohort study. Eur J Cancer 2010, 46(10):1873-1881.

20. Stroup DF, Berlin JA, Morton SC, Olkin I, Williamson GD, Rennie D, Moher D, Becker BJ, Sipe TA, Thacker SB: Meta-analysis of observational studies in epidemiology: a proposal for reporting. Meta-analysis Of Observational Studies in Epidemiology (MOOSE) group. JAMA: j Am Med Assoc 2000, 283(15):2008-2012.

21. Wells GA, Shea B, O'Connell D, Peterson J, Welch V, Losos M, Tugwell P: The Newcastle-Ottawa Scale (NOS) for assessing the quality of nonrandomised studies in meta-analyses. Ottawa, Canada: Department of Epidemiology and Community Medicine, University of Ottawa; 2000. http://www.ohri.ca/ programs/clinical_epidemiology/oxford.asp (accessed date Mar 16 2014).

22. Orsini N, Bellocco RSG: Generalized least squares for trend estimation of summarized dose-response data. Stata J 2006, 6:40-57.

23. Greenland S, Longnecker MP: Methods for trend estimation from summarized dose-response data, with applications to meta-analysis. Am J Epidemiol 1992, 135:1301-1309.

24. Greenland S: Quantitative methods in the review of epidemiologic literature. Epidemiol Rev 1987, 9:1-30.

25. Ishitani K, Lin J, Manson JE, Buring JE, Zhang SM: Caffeine consumption and the risk of breast cancer in a large prospective cohort of women Arch Intern Med 2008, 168(18):2022-2031. 
26. Lee KJ, Inoue M, Otani T, Iwasaki M, Sasazuki S, Tsugane S: Coffee consumption and risk of colorectal cancer in a population-based prospective cohort of Japanese men and women. Int J Cancer 2007, 121(6):1312-1318.

27. Nechuta S, Shu XO, Li HL, Yang G, Ji BT, Xiang YB, Cai H, Chow WH, Gao YT, Zheng W: Prospective cohort study of tea consumption and risk of digestive system cancers: results from the Shanghai Women's Health Study. Am J Clin Nutr 2012, 96:1056-1063. 10/12 edn; 2012

28. Su LJ, Arab L: Tea consumption and the reduced risk of colon cancer results from a national prospective cohort study. Public Health Nutr 2002, 5(3):419-425.

29. Dominianni $C$, Huang WY, Berndt S, Hayes RB, Ahn J: Prospective study of the relationship between coffee and tea with colorectal cancer risk: the PLCO Cancer Screening Trial. Br J Cancer 2013, 109(5):1352-1359.

30. Montague JA, Butler LM, Wu AH, Genkinger JM, Koh WP, Wong AS, Wang R, Yuan JM, Yu MC: Green and black tea intake in relation to prostate cancer risk among Singapore Chinese. Cancer Causes Control 2012, 23(10):1635-1641.

31. Oba S, Shimizu N, Nagata C, Shimizu H, Kametani M, Takeyama N, Ohnuma T, Matsushita S: The relationship between the consumption of meat, fat, and coffee and the risk of colon cancer: a prospective study in Japan. Cancer lett 2006, 244(2):260-267.

32. Aune D, Greenwood DC, Chan DS, Vieira R, Vieira AR, Navarro Rosenblatt DA, Cade JE, Burley VJ, Norat T: Body mass index, abdominal fatness and pancreatic cancer risk: a systematic review and non-linear dose-response meta-analysis of prospective studies. Ann oncol: official j the Eur Soc Med Oncol / ESMO 2012, 23(4):843-852.

33. Dai Q, Shu XO, Li H, Yang G, Shrubsole MJ, Cai H, Ji B, Wen W, Franke A, Gao YT, Zheng W: Is green tea drinking associated with a later onset of breast cancer? Ann Epidemiol 2010, 20(1):74-81.

34. DerSimonian R, Laird N: Meta-analysis in clinical trials. Control Clin Trials 1986, 7(3):177-188.

35. Higgins JPT, Green S: Cochrane Handbook for Systematic Reviews of Interventions, Version 5.1.0 [updated March 2011]. The Cochrane Collaboration 2011. Available from www.cochrane-handbook.org.

36. Larsson SC, Orsini N: Coffee consumption and risk of stroke: a doseresponse meta-analysis of prospective studies. Am J Epidemiol 2011, 174(9):993-1001.

37. Larsson SC, Orsini N, Wolk A: Dietary magnesium intake and risk of stroke: a meta-analysis of prospective studies. Am J Clin Nutr 2012, 95(2):362-366.

38. Simons CC, Leurs LJ, Weijenberg MP, Schouten LJ, Goldbohm RA, van den Brandt PA: Fluid intake and colorectal cancer risk in the Netherlands Cohort Study. Nutr Cancer 2010, 62(3):307-321.

39. Michels KB, Willett WC, Fuchs CS, Giovannucci E: Coffee, tea, and caffeine consumption and incidence of colon and rectal cancer. J Natl Cancer Inst 2005, 97(4):282-292.

40. Hartman TJ, Tangrea JA, Pietinen P, Malila N, Virtanen M, Taylor PR, Albanes D: Tea and coffee consumption and risk of colon and rectal cancer in middle-aged Finnish men. Nutr Cancer 1998, 31(1):41-48.

41. Zheng W, Doyle TJ, Kushi LH, Sellers TA, Hong CP, Folsom AR: Tea consumption and cancer incidence in a prospective cohort study of postmenopausal women. Am J Epidemiol 1996, 144(2):175-182.

42. Galanis DJ, Kolonel LN, Lee J, Nomura A: Intakes of selected foods and beverages and the incidence of gastric cancer among the Japanese residents of Hawaii: a prospective study. Int J Epidemio/ 1998, 27(2):173-180.

43. Inoue M, Sasazuki S, Wakai K, Suzuki T, Matsuo K, Shimazu T, Tsuji I, Tanaka K, Mizoue T, Nagata C, Tamakoshi A, Sawada N, Tsugane S: Green tea consumption and gastric cancer in Japanese: a pooled analysis of six cohort studies. Gut 2009, 58(10):1323-1332.

44. Suzuki Y, Tsubono Y, Nakaya N, Koizumi Y, Shibuya D, Tsuji I: Green tea and the risk of colorectal cancer: pooled analysis of two prospective studies in Japan. J Epidemiol 2005, 15(4):118-124.

45. Terry $P$, Wolk A: Tea consumption and the risk of colorectal cancer in Sweden. Nutr Cancer 2001, 39(2):176-179.

46. Higgins JP, Thompson SG: Quantifying heterogeneity in a meta-analysis. Stat Med 2002, 21(11):1539-1558.

47. Begg CB, Mazumdar M: Operating characteristics of a rank correlation test for publication bias. Biometrics 1994, 50(4):1088-1101.

48. Egger M, Davey Smith G, Schneider M, Minder C: Bias in meta-analysis detected by a simple, graphical test. Br Med J 1997, 315(7109):629-634.
49. Adebamowo CA, Cho E, Sampson L, Katan MB, Spiegelman D, Willett WC, Holmes MD: Dietary flavonols and flavonol-rich foods intake and the risk of breast cancer. Int J Cancer 2005, 114(4):628-633.

50. Allen NE, Sauvaget C, Roddam AW, Appleby P, Nagano J, Suzuki G, Key TJ, Koyama K: A prospective study of diet and prostate cancer in Japanese men. Cancer Causes Control 2004, 15(9):911-920.

51. Key TJ, Sharp GB, Appleby PN, Beral V, Goodman MT, Soda M, Mabuchi K: Soya foods and breast cancer risk: a prospective study in Hiroshima and Nagasaki. Japan. Br J Cancer 1999, 81(7):1248-1256.

52. Sauvaget C, Lagarde F, Nagano J, Soda M, Koyama K, Kodama K: Lifestyle factors, radiation and gastric cancer in atomic-bomb survivors (Japan). Cancer Causes Control 2005, 16(7):773-780.

53. Ellison LF: Tea and other beverage consumption and prostate cancer risk: a Canadian retrospective cohort study. Eur J Cancer Prev 2000, 9(2):125-130.

54. Hirvonen T, Mennen LI, de Bree A, Castetbon K, Galan P, Bertrais S, Arnault $\mathrm{N}$, Hercberg S: Consumption of antioxidant-rich beverages and risk for breast cancer in French women. Ann Epidemiol 2006, 16(7):503-508.

55. Bhoo Pathy N, Peeters P, van Gils C, Beulens JW, van der Graaf $Y$, Bueno-deMesquita B, Bulgiba A, Uiterwaal CS: Coffee and tea intake and risk of breast cancer. Breast Cancer Res Treat 2010, 121(2):461-467.

56. Boggs DA, Palmer JR, Stampfer MJ, Spiegelman D, Adams-Campbell LL, Rosenberg $L$ : Tea and coffee intake in relation to risk of breast cancer in the Black Women's Health Study. Cancer Causes Control 2010, 21(11):1941-1948.

57. Fagherazzi G, Touillaud MS, Boutron-Ruault MC, Clavel-Chapelon F, Romieu I: No association between coffee, tea or caffeine consumption and breast cancer risk in a prospective cohort study. Public Health Nutr 2011, 14(7):1315-1320.

58. Ganmaa D, Willett WC, Li TY, Feskanich D, van Dam RM, Lopez-Garcia E, Hunter DJ, Holmes MD: Coffee, tea, caffeine and risk of breast cancer: a 22-year follow-up. Int J Cancer 2008, 122(9):2071-2076.

59. Goldbohm RA, Hertog MG, Brants HA, van Poppel G, van den Brandt PA: Consumption of black tea and cancer risk: a prospective cohort study. J Natl Cancer Inst 1996, 88(2):93-100.

60. Iwasaki M, Inoue M, Sasazuki S, Sawada N, Yamaji T, Shimazu T, Willett WC Tsugane S, Jphc FT: Green tea drinking and subsequent risk of breast cancer in a population-based cohort of Japanese women. Breast Cancer Res 2010, 12(5):R88.

61. Larsson SC, Bergkvist L, Wolk A: Coffee and black tea consumption and risk of breast cancer by estrogen and progesterone receptor status in a Swedish cohort. Cancer Causes Control 2009, 20(10):2039-2044.

62. Michels KB, Holmberg L, Bergkvist L, Wolk A: Coffee, tea, and caffeine consumption and breast cancer incidence in a cohort of Swedish women. Ann Epidemiol 2002, 12(1):21-26.

63. Suzuki Y, Tsubono Y, Nakaya N, Koizumi Y, Tsuji I: Green tea and the risk of breast cancer: pooled analysis of two prospective studies in Japan. $\mathrm{Br} J$ Cancer 2004, 90(7):1361-1363.

64. Inoue M, Kurahashi N, Iwasaki M, Shimazu T, Tanaka Y, Mizokami M, Tsugane S: Effect of coffee and green tea consumption on the risk of liver cancer: cohort analysis by hepatitis virus infection status. Cancer Epidemiol Biomarkers Prev 2009, 18(6):1746-1753.

65. Ui A, Kuriyama S, Kakizaki M, Sone T, Nakaya N, Ohmori-Matsuda K, Hozawa A, Nishino Y, Tsuji I: Green tea consumption and the risk of liver cancer in Japan: the Ohsaki Cohort study. Cancer Causes Control 2009, 20(10):1939-1945.

66. Kikuchi N, Ohmori K, Shimazu T, Nakaya N, Kuriyama S, Nishino Y, Tsubono $Y, T$ suji I: No association between green tea and prostate cancer risk in Japanese men: the Ohsaki Cohort Study. Br J Cancer 2006, 95(3):371-373.

67. Kurahashi N, Sasazuki S, Iwasaki M, Inoue M, Tsugane S: Green tea consumption and prostate cancer risk in Japanese men: a prospective study. Am J Epidemiol 2008, 167(1):71-77.

68. Shafique K, McLoone P, Qureshi K, Leung H, Hart C, Morrison DS: Tea consumption and the risk of overall and grade specific prostate cancer: a large prospective cohort study of Scottish men. Nutr Cancer 2012, 64(6):790-797.

69. Geybels MS, Verhage BA, Arts IC, van Schooten FJ, Goldbohm RA, van den Brandt PA: Dietary flavonoid intake, black tea consumption, and risk of overall and advanced stage prostate cancer. Am J Epidemiol 2013, 177(12):1388-1398

70. Kang H, Rha SY, Oh KW, Nam CM: Green tea consumption and stomach cancer risk: a meta-analysis. Epidemiol Health 2010, 32:e2010001. 
71. Myung SK, Bae WK, Oh SM, Kim Y, Ju W, Sung J, Lee YJ, Ko JA, Song Jl, Choi $\mathrm{HJ}$ : Green tea consumption and risk of stomach cancer: a meta-analysis of epidemiologic studies. Int J Cancer 2009, 124(3):670-677.

72. Seely D, Mills EJ, Wu P, Verma S, Guyatt GH: The effects of green tea consumption on incidence of breast cancer and recurrence of breast cancer: a systematic review and meta-analysis. Integr Cancer Ther 2005 , 4(2):144-155.

73. Sun CL, Yuan JM, Koh WP, Yu MC: Green tea, black tea and breast cancer risk: a meta-analysis of epidemiological studies. Carcinogenesis 2006, 27(7):1310-1315.

74. Zhang X, Albanes D, Beeson WL, van den Brandt PA, Buring JE, Flood A, Freudenheim JL, Giovannucci EL, Goldbohm RA, Jaceldo-Siegl K, Jacobs EJ, Krogh V, Larsson SC, Marshall JR, McCullough ML, Miller AB, Robien K, Rohan TE, Schatzkin A, Sieri S, Spiegelman D, Virtamo J, Wolk A, Willett WC, Zhang SM, Smith-Warner SA: Risk of colon cancer and coffee, tea, and sugarsweetened soft drink intake: pooled analysis of prospective cohort studies. J Natl Cancer Inst 2010, 102(11):771-783.

75. Zhou Y, Li N, Zhuang W, Liu G, Wu T, Yao X, Du L, Wei M, Wu X: Green tea and gastric cancer risk: meta-analysis of epidemiologic studies. Asia Pac $J$ Clin Nutr 2008, 17(1):159-165.

76. Platet N, Cathiard AM, Gleizes M, Garcia M: Estrogens and their receptors in breast cancer progression: a dual role in cancer proliferation and invasion. Crit Rev Oncol Hematol 2004, 51(1):55-67.

77. Wu AH, Arakawa K, Stanczyk FZ, Van Den Berg D, Koh WP, Yu MC: Tea and circulating estrogen levels in postmenopausal Chinese women in Singapore. Carcinogenesis 2005, 26(5):976-980

doi:10.1186/1471-2407-14-197

Cite this article as: Yu et al:: Tea consumption and the risk of five major cancers: a dose-response meta-analysis of prospective studies. BMC

Cancer 2014 14:197.

\section{Submit your next manuscript to BioMed Central and take full advantage of:}

- Convenient online submission

- Thorough peer review

- No space constraints or color figure charges

- Immediate publication on acceptance

- Inclusion in PubMed, CAS, Scopus and Google Scholar

- Research which is freely available for redistribution

Submit your manuscript at www.biomedcentral.com/submit

C Biomed Central 